\title{
Substituent Effect on Triplet State Aromaticity of Benzene
}

\author{
Marija Baranac-Stojanović \\ University of Belgrade - Faculty of Chemistry, Studentski trg 12-16, P.O.Box 158, 11000 Belgrade, Serbia
}

Supporting Information

\section{Table of Contents}

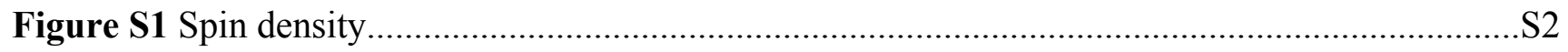

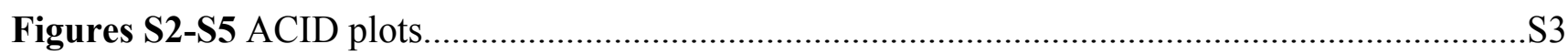

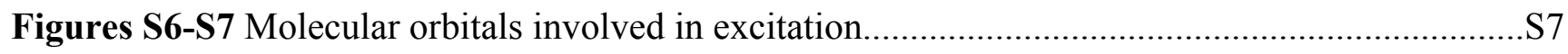

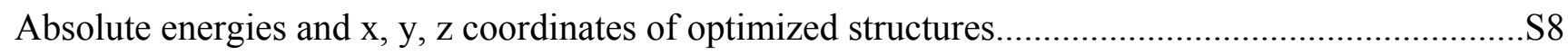



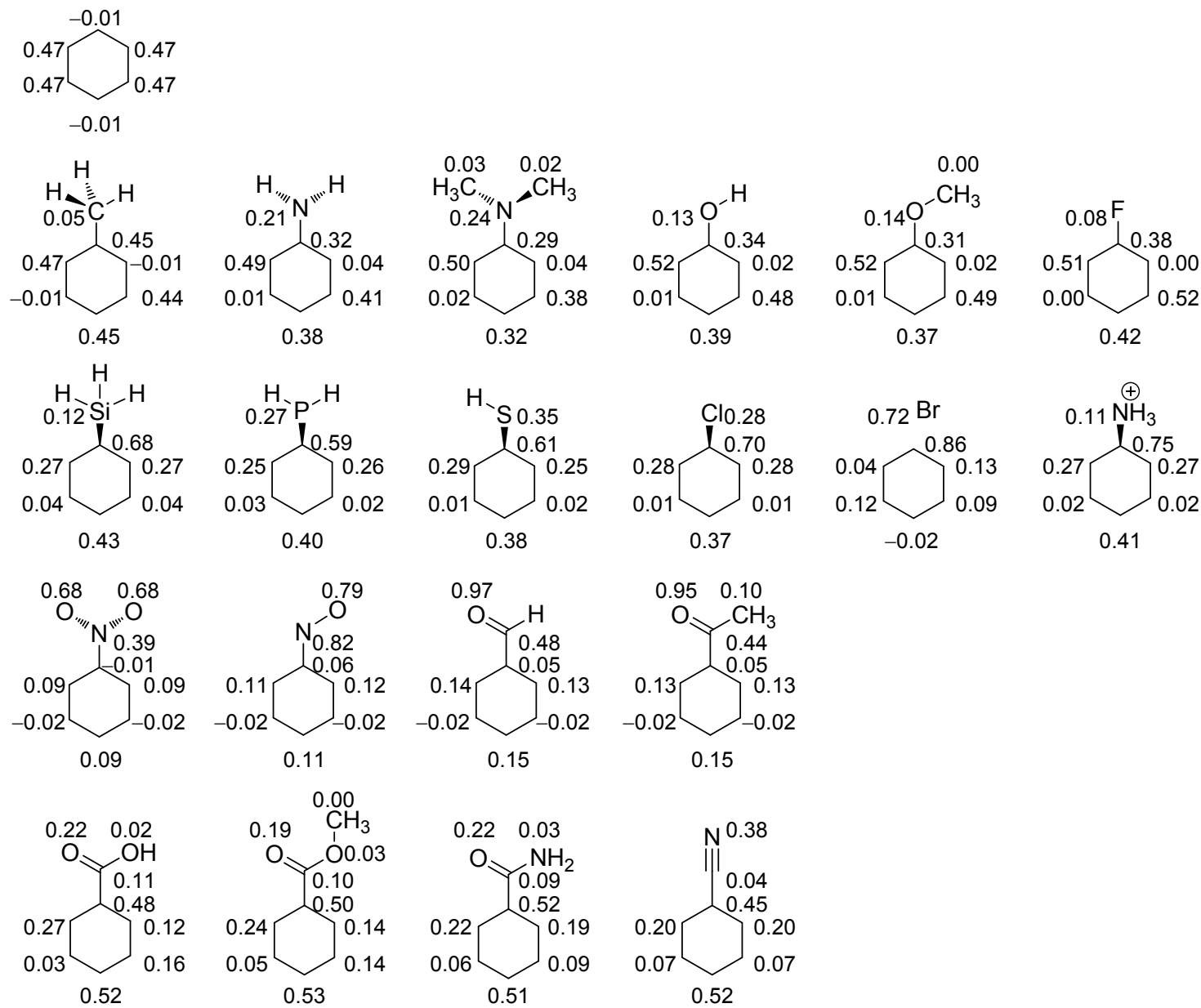

Figure S1. Spin densities at heavy atoms obtained by the Hirshfeld population analysis. 


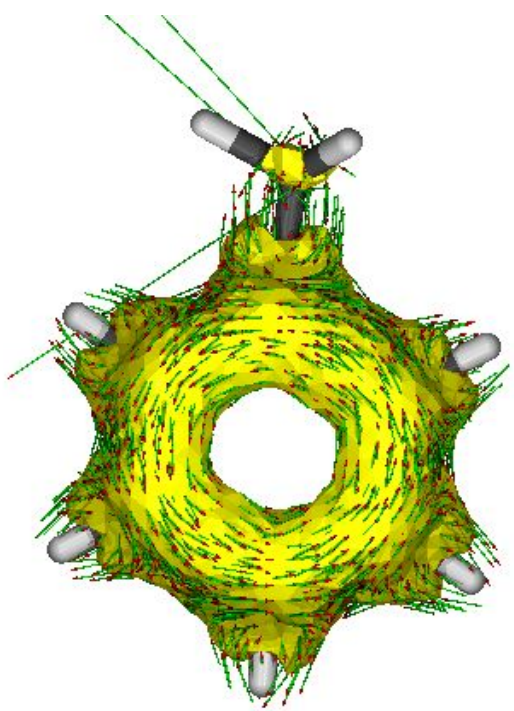

$\mathrm{R}=\mathrm{CH}_{3}$

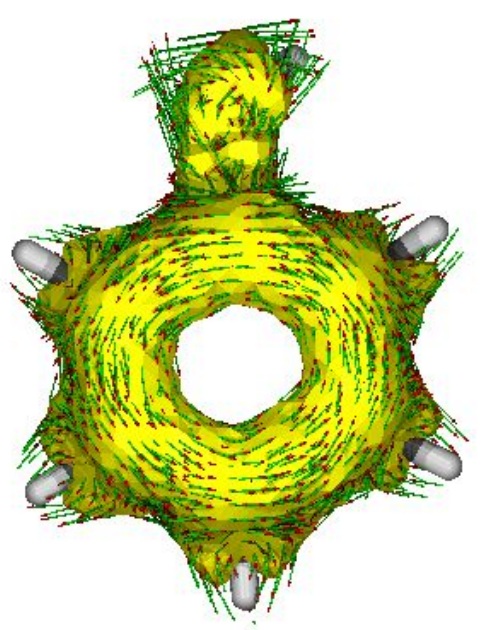

$\mathrm{R}=\mathrm{OH}$

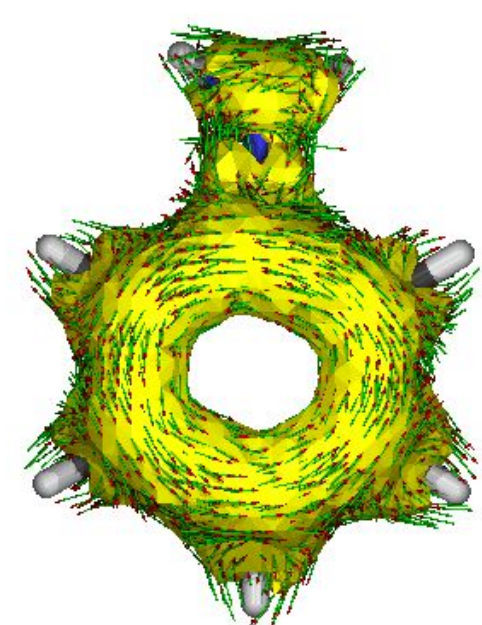

$\mathrm{R}=\mathrm{NH}_{2}$

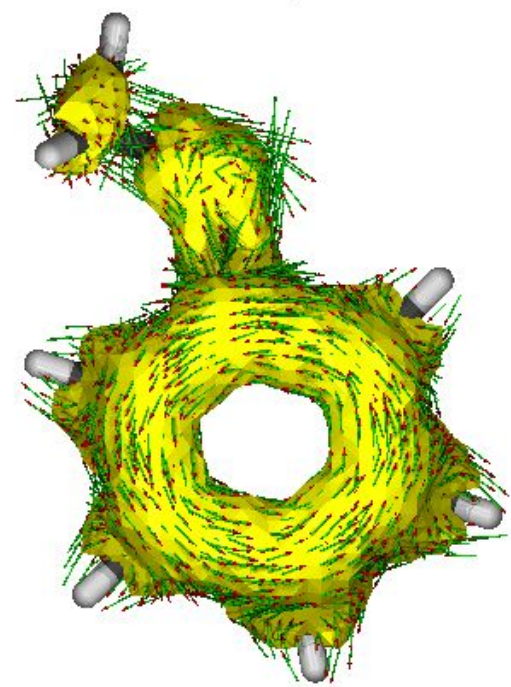

$\mathrm{R}=\mathrm{OCH}_{3}$

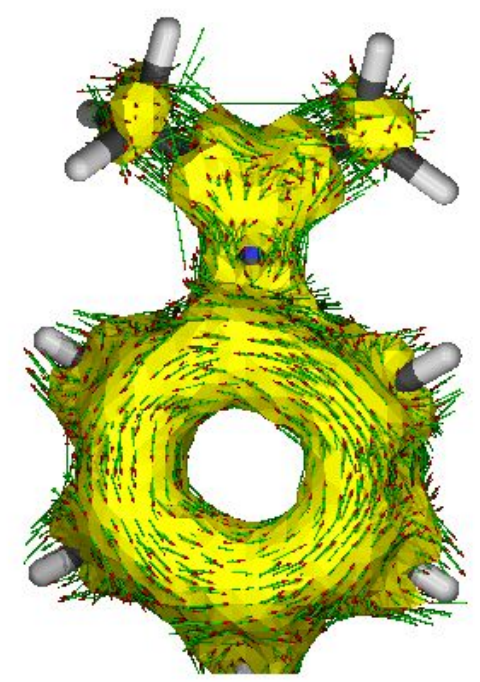

$\mathrm{R}=\mathrm{N}\left(\mathrm{CH}_{3}\right)_{2}$

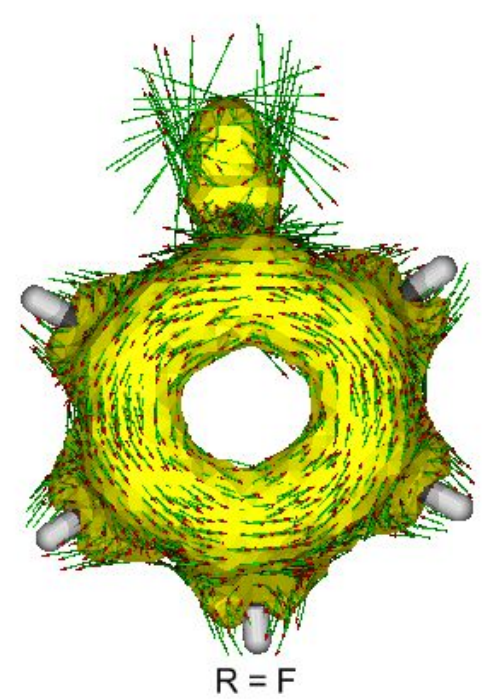

Figure S2. ACID plots of benzenes substituted with $\sigma$ - and $\pi$-electron donating groups. Clockwise circulation is diatropic and counterclockwise circulation is paratropic. 


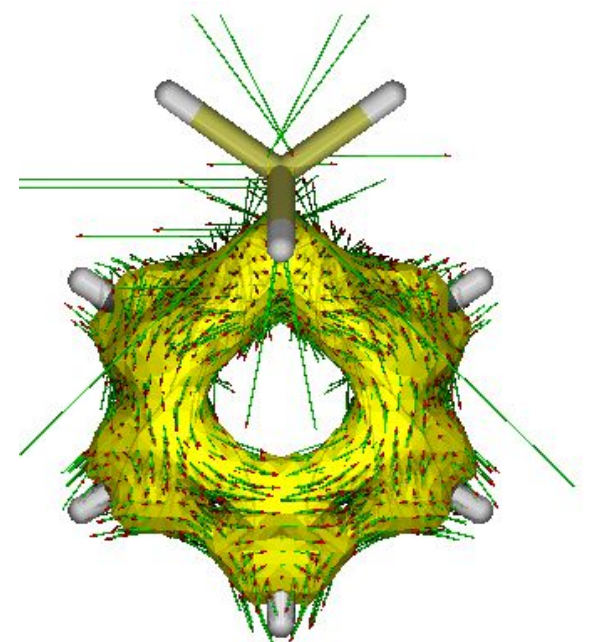

$\mathrm{R}=\mathrm{SiH}_{3}$

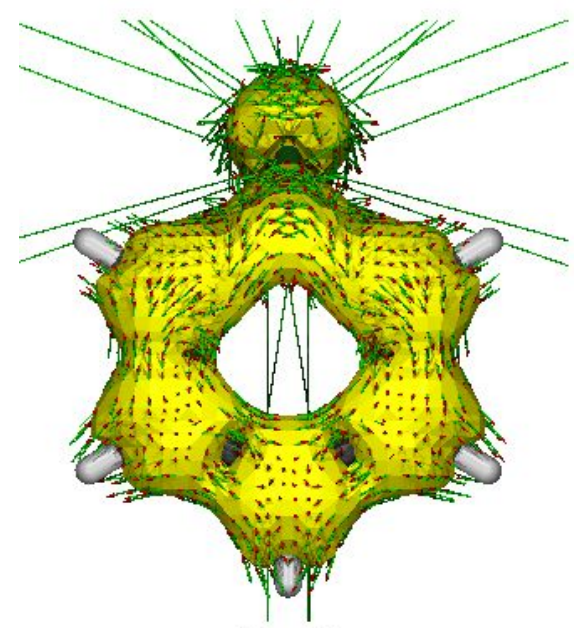

$\mathrm{R}=\mathrm{Cl}$

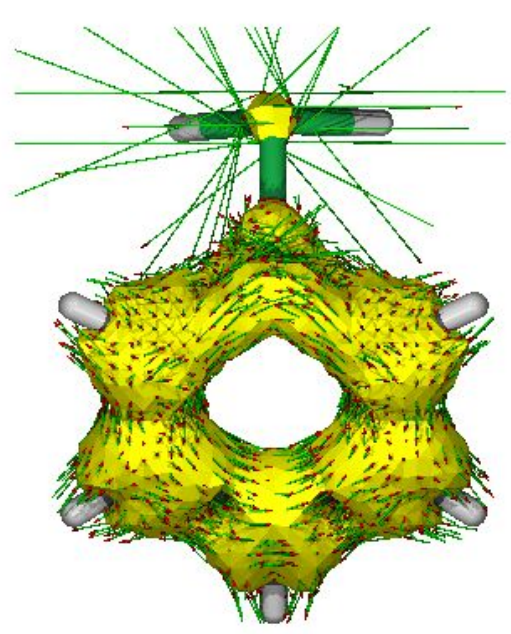

$\mathrm{R}=\mathrm{PH}_{2}$

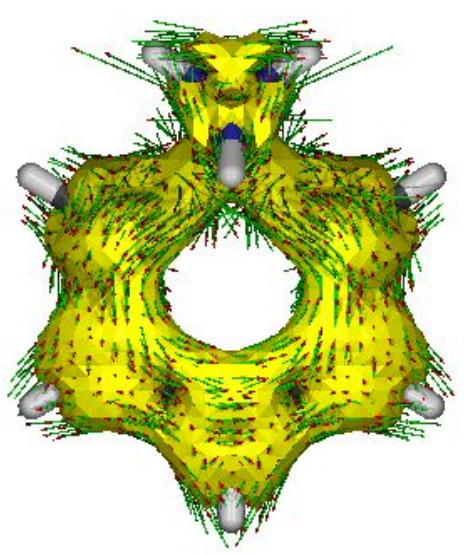

$\mathrm{R}=\mathrm{NH}_{3}{ }^{+}$

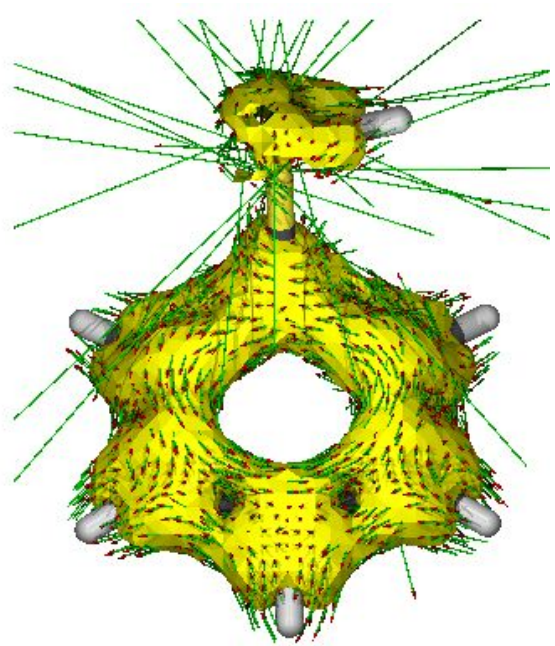

$\mathrm{R}=\mathrm{SH}$

Figure S3. ACID plots of benzenes substituted with groups containing the third row elements and $\sigma$-electron accepting $\mathrm{NH}_{3}{ }^{+}$group. Clockwise circulation is diatropic and counterclockwise circulation is paratropic. 

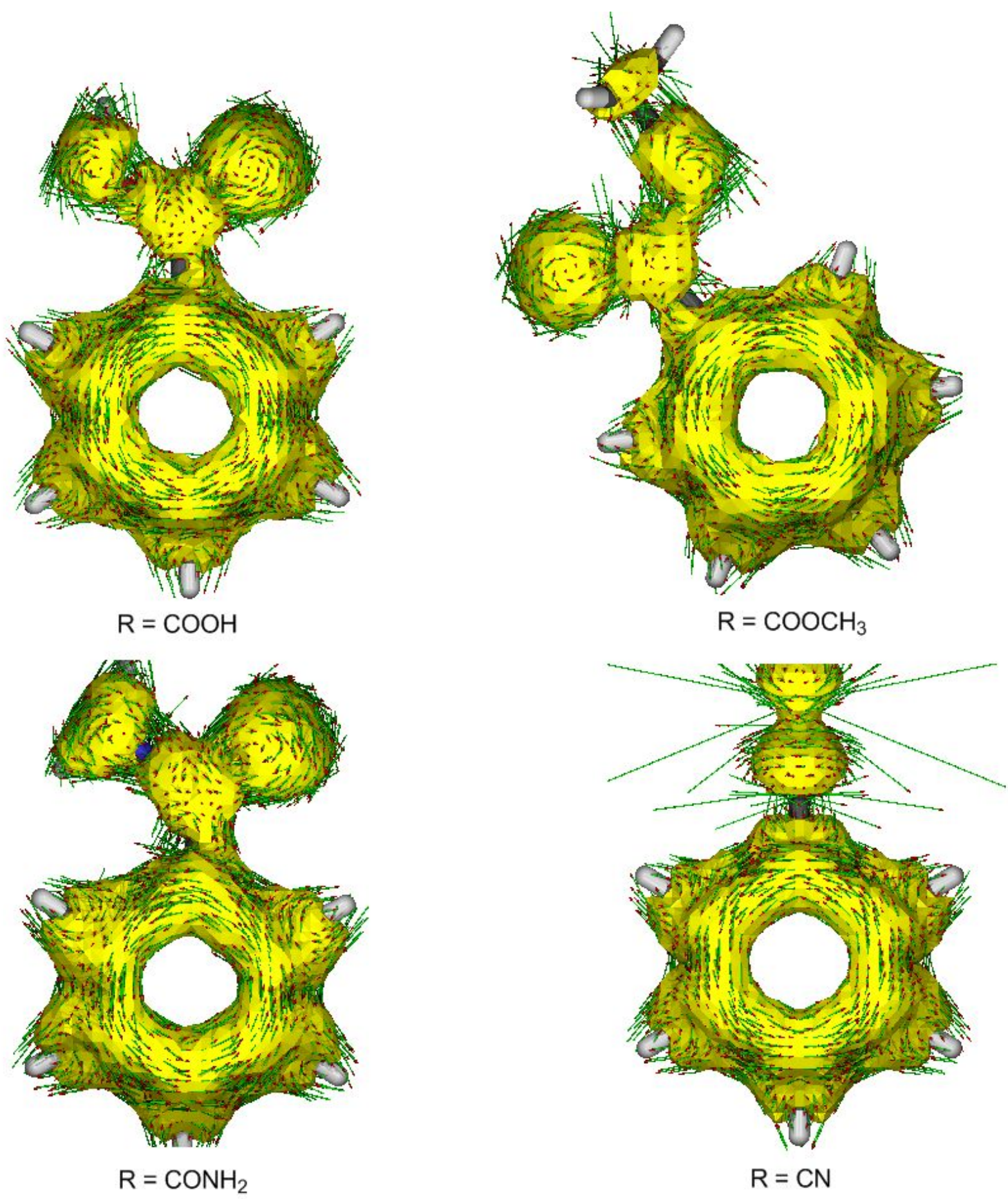

Figure S4. ACID plots of benzenes substituted with $\pi$-electron accepting groups. Clockwise circulation is diatropic and counterclockwise circulation is paratropic. 

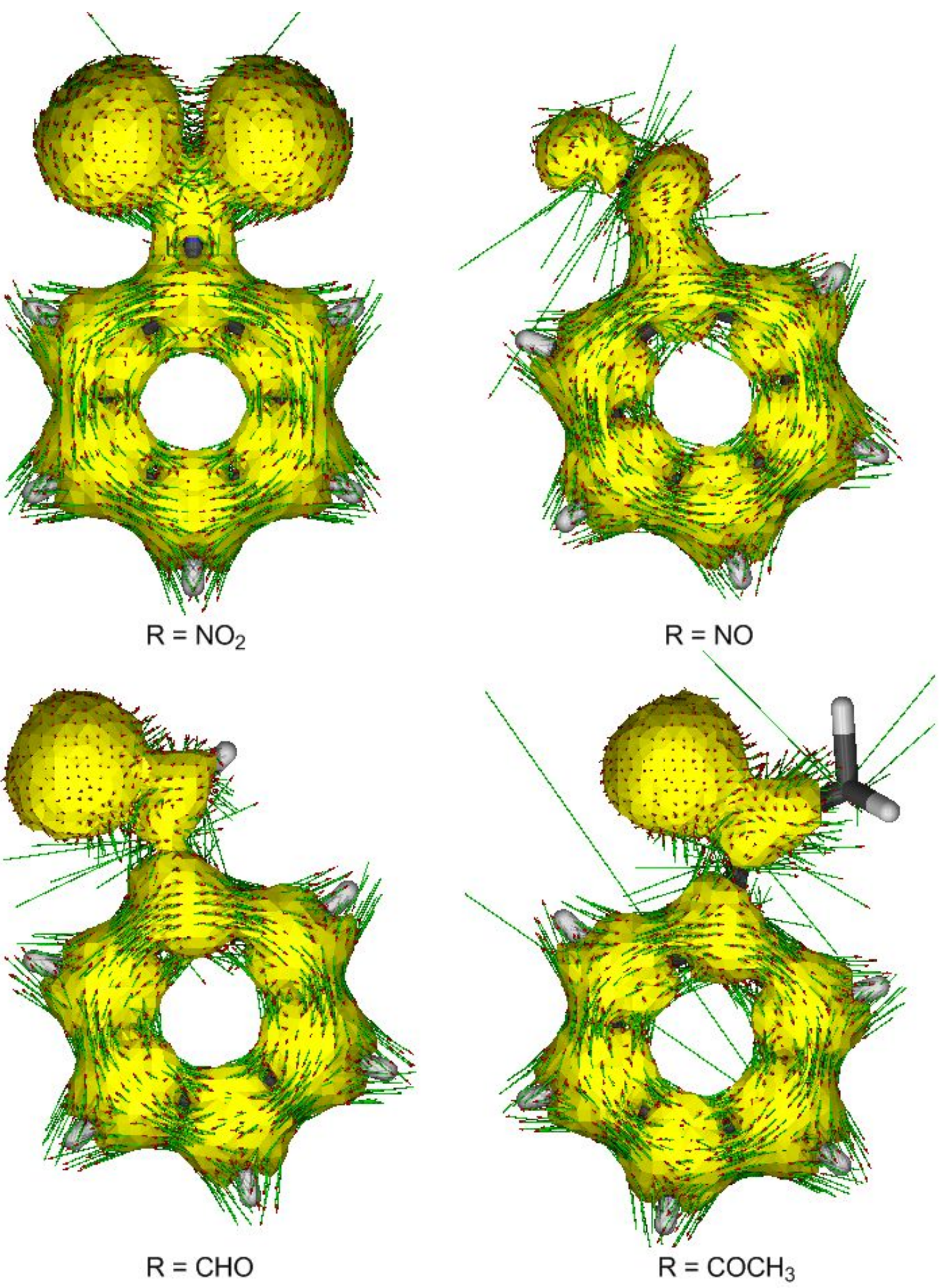

Figure S5. ACID plots of benzenes substituted with $\pi$-electron accepting groups. Clockwise circulation is diatropic and counterclockwise circulation is paratropic. 


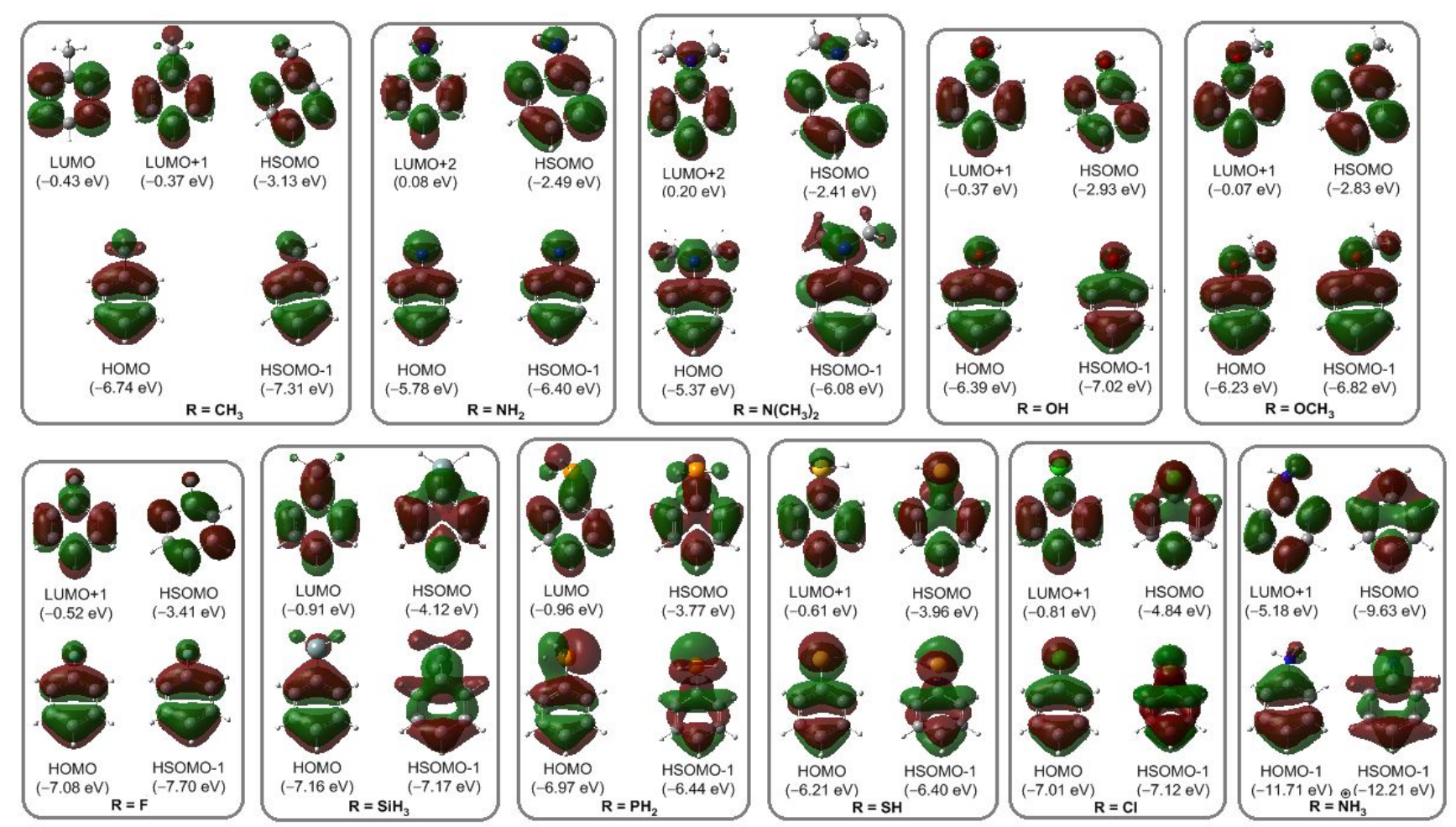

Figure S6. Molecular orbitals involved in the most important $S_{0} \rightarrow T_{1}$ excitation of compounds containing $\sigma$ - or $\pi$-electron donating substituents and electron-accepting $\mathrm{NH}_{3}{ }^{+}$group, at an isovalue of 0.03 a.u.
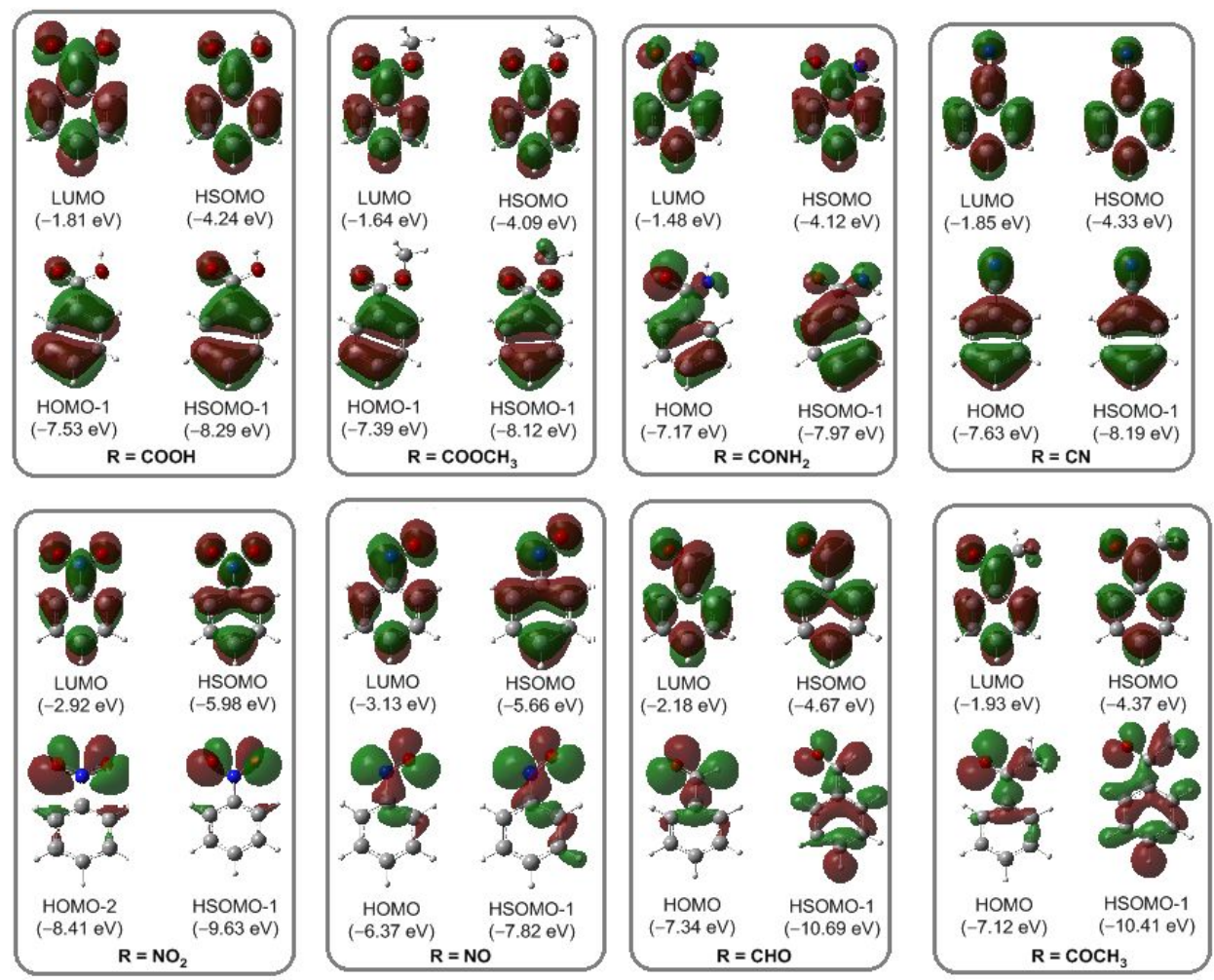

Figure S7. Molecular orbitals involved in the most important $S_{0} \rightarrow T_{1}$ excitation of compounds containing $\pi$-electron accepting substituents, at an isovalue of 0.03 a.u. 


\section{Absolute energies (a.u.) and $x, y, z$ coordinates of optimized structures}

\section{Benzene $\mathrm{S}_{0}$}

$\mathrm{E}=-232.3112416$ a.u.

\begin{tabular}{|c|c|c|c|c|c|}
\hline \multirow{2}{*}{$\begin{array}{l}\text { Center } \\
\text { Number }\end{array}$} & \multirow{2}{*}{$\begin{array}{l}\text { Atomic } \\
\text { Number }\end{array}$} & \multirow{2}{*}{$\begin{array}{c}\text { Atomic } \\
\text { Type }\end{array}$} & \multicolumn{3}{|c|}{ Coordinates (Angstroms) } \\
\hline & & & $\mathrm{X}$ & $\mathrm{Y}$ & Z \\
\hline 1 & 6 & 0 & 0.000000 & 1.394301 & 0.000000 \\
\hline 2 & 6 & 0 & 1.207500 & 0.697151 & 0.000000 \\
\hline 3 & 6 & 0 & 1.207500 & -0.697151 & 0.000000 \\
\hline 4 & 6 & 0 & 0.000000 & -1.394301 & 0.000000 \\
\hline 5 & 6 & 0 & -1.207500 & -0.697151 & 0.000000 \\
\hline 6 & 6 & 0 & -1.207500 & 0.697151 & 0.000000 \\
\hline 7 & 1 & 0 & 0.000000 & 2.478574 & 0.000000 \\
\hline 8 & 1 & 0 & 2.146508 & 1.239287 & 0.000000 \\
\hline 9 & 1 & 0 & 2.146508 & -1.239287 & 0.000000 \\
\hline 10 & 1 & 0 & 0.000000 & -2.478574 & 0.000000 \\
\hline 11 & 1 & 0 & -2.146508 & -1.239287 & 0.000000 \\
\hline 12 & 1 & 0 & -2.146508 & 1.239287 & 0.000000 \\
\hline
\end{tabular}

\section{Benzene $\mathrm{T}_{1}$}

$\mathrm{E}=-232.1699199$ a.u.

\begin{tabular}{|c|c|c|c|c|c|}
\hline \multirow{2}{*}{$\begin{array}{l}\text { Center } \\
\text { Number }\end{array}$} & \multirow{2}{*}{$\begin{array}{l}\text { Atomic } \\
\text { Number }\end{array}$} & \multirow{2}{*}{$\begin{array}{c}\text { Atomic } \\
\text { Type }\end{array}$} & \multicolumn{3}{|c|}{ Coordinates (Angstroms) } \\
\hline & & & $\mathrm{X}$ & $Y$ & Z \\
\hline 1 & 6 & 0 & 0.000000 & 1.207797 & 0.759712 \\
\hline 2 & 6 & 0 & 0.000000 & 0.000000 & 1.441395 \\
\hline 3 & 6 & 0 & 0.000000 & -1.207797 & 0.759712 \\
\hline 4 & 6 & 0 & 0.000000 & -1.207797 & -0.759712 \\
\hline 5 & 6 & 0 & 0.000000 & 0.000000 & -1.441395 \\
\hline 6 & 6 & 0 & 0.000000 & 1.207797 & -0.759712 \\
\hline 7 & 1 & 0 & 0.000000 & 2.152534 & 1.287125 \\
\hline 8 & 1 & 0 & 0.000000 & 0.000000 & 2.526694 \\
\hline 9 & 1 & 0 & 0.000000 & -2.152534 & 1.287125 \\
\hline 10 & 1 & 0 & 0.000000 & -2.152534 & -1.287125 \\
\hline 11 & 1 & 0 & 0.000000 & 0.000000 & -2.526694 \\
\hline 12 & 1 & 0 & 0.000000 & 2.152534 & -1.287125 \\
\hline
\end{tabular}




\begin{tabular}{|c|c|c|c|c|c|}
\hline \multicolumn{6}{|c|}{$\begin{array}{l}\mathbf{R}=\mathbf{C H}_{\mathbf{3}} \mathrm{S}_{0} \\
\mathrm{E}=-271.6388137 \text { a.u. }\end{array}$} \\
\hline \multirow{2}{*}{$\begin{array}{l}\text { Center } \\
\text { Number }\end{array}$} & \multirow{2}{*}{$\begin{array}{l}\text { Atomic } \\
\text { Number }\end{array}$} & \multirow{2}{*}{$\begin{array}{c}\text { Atomic } \\
\text { Type }\end{array}$} & \multicolumn{3}{|c|}{ Coordinates (Angstroms) } \\
\hline & & & $\mathrm{X}$ & $Y$ & Z \\
\hline 1 & 6 & 0 & -0.007555 & -1.199548 & 1.203415 \\
\hline 2 & 6 & 0 & -0.007555 & 0.194230 & 1.200484 \\
\hline 3 & 6 & 0 & -0.004273 & 0.913135 & 0.000000 \\
\hline 4 & 6 & 0 & -0.007555 & 0.194230 & -1.200484 \\
\hline 5 & 6 & 0 & -0.007555 & -1.199548 & -1.203415 \\
\hline 6 & 6 & 0 & -0.006636 & -1.902681 & 0.000000 \\
\hline 7 & 1 & 0 & -0.012150 & -1.736113 & 2.146085 \\
\hline 8 & 1 & 0 & -0.012602 & 0.732057 & 2.143638 \\
\hline 9 & 1 & 0 & -0.012602 & 0.732057 & -2.143638 \\
\hline 10 & 1 & 0 & -0.012150 & -1.736113 & -2.146085 \\
\hline 11 & 1 & 0 & -0.009469 & -2.986956 & 0.000000 \\
\hline 12 & 6 & 0 & 0.029022 & 2.423022 & 0.000000 \\
\hline 13 & 1 & 0 & 1.060711 & 2.792130 & 0.000000 \\
\hline 14 & 1 & 0 & -0.464553 & 2.832953 & -0.884499 \\
\hline 15 & 1 & 0 & -0.464553 & 2.832953 & 0.884499 \\
\hline
\end{tabular}

\section{$\mathbf{R}=\mathbf{C H}_{\mathbf{3}} \mathrm{T}_{1}$ \\ $\mathrm{E}=-271.5030272$ a.u.}

\begin{tabular}{|c|c|c|c|c|c|}
\hline \multirow{2}{*}{$\begin{array}{l}\text { Center } \\
\text { Number }\end{array}$} & \multirow{2}{*}{$\begin{array}{l}\text { Atomic } \\
\text { Number }\end{array}$} & \multirow{2}{*}{$\begin{array}{l}\text { Atomic } \\
\text { Type }\end{array}$} & \multicolumn{3}{|c|}{ Coordinates (Angstroms) } \\
\hline & & & $\mathrm{X}$ & $\mathrm{Y}$ & Z \\
\hline 1 & 6 & 0 & -1.339444 & -1.082552 & 0.000000 \\
\hline 2 & 6 & 0 & -1.235172 & 0.296005 & 0.000000 \\
\hline 3 & 6 & 0 & 0.000000 & 0.941723 & 0.000000 \\
\hline 4 & 6 & 0 & 1.260974 & 0.082772 & 0.000000 \\
\hline 5 & 6 & 0 & 1.155015 & -1.302155 & 0.000000 \\
\hline 6 & 6 & 0 & -0.082614 & -1.922438 & 0.000000 \\
\hline 7 & 1 & 0 & -2.303040 & -1.574292 & 0.000000 \\
\hline 8 & 1 & 0 & -2.139355 & 0.898483 & 0.000000 \\
\hline 9 & 1 & 0 & 2.224891 & 0.576320 & 0.000000 \\
\hline 10 & 1 & 0 & 2.056460 & -1.906817 & 0.000000 \\
\hline 11 & 1 & 0 & -0.168229 & -3.001476 & 0.000000 \\
\hline 12 & 6 & 0 & 0.174674 & 2.415347 & 0.000000 \\
\hline 13 & 1 & 0 & 0.754391 & 2.745357 & 0.876697 \\
\hline 14 & 1 & 0 & 0.754391 & 2.745357 & -0.876697 \\
\hline 15 & 1 & 0 & -0.780104 & 2.944855 & 0.000000 \\
\hline
\end{tabular}




\begin{tabular}{|c|c|c|c|c|c|}
\hline $\begin{array}{l}\mathbf{R}=\mathbf{N H}_{\mathbf{2}} \mathrm{S} \\
\mathrm{E}=-287.6\end{array}$ & 74 a.u. & & & & \\
\hline Center & Atomic & Atomic & Coor & inates (Ang & troms) \\
\hline Number & Number & Type & $\mathrm{X}$ & $Y$ & Z \\
\hline 1 & 6 & 0 & -0.003385 & -1.170662 & 1.200866 \\
\hline 2 & 6 & 0 & -0.003385 & 0.220540 & 1.206220 \\
\hline 3 & 6 & 0 & -0.002114 & 0.937027 & 0.000000 \\
\hline 4 & 6 & 0 & -0.003385 & 0.220540 & -1.206220 \\
\hline 5 & 6 & 0 & -0.003385 & -1.170662 & -1.200866 \\
\hline 6 & 6 & 0 & -0.003246 & -1.879746 & 0.000000 \\
\hline 7 & 1 & 0 & -0.002925 & -1.703712 & 2.145552 \\
\hline 8 & 1 & 0 & -0.008922 & 0.759878 & 2.148457 \\
\hline 9 & 1 & 0 & -0.008922 & 0.759878 & -2.148457 \\
\hline 10 & 1 & 0 & -0.002925 & -1.703712 & -2.145552 \\
\hline 11 & 1 & 0 & -0.002727 & -2.963107 & 0.000000 \\
\hline 12 & 7 & 0 & -0.059499 & 2.334027 & 0.000000 \\
\hline 13 & 1 & 0 & 0.278153 & 2.785185 & 0.837363 \\
\hline 14 & 1 & 0 & 0.278153 & 2.785185 & -0.837363 \\
\hline
\end{tabular}

\section{$\mathbf{R}=\mathbf{N H}_{\mathbf{2}} \mathrm{T}_{1}$ \\ $\mathrm{E}=-287.5601692$ a.u.}

\begin{tabular}{|c|c|c|c|c|c|}
\hline \multirow{2}{*}{$\begin{array}{l}\text { Center } \\
\text { Number }\end{array}$} & \multirow{2}{*}{$\begin{array}{l}\text { Atomic } \\
\text { Number }\end{array}$} & \multirow{2}{*}{$\begin{array}{c}\text { Atomic } \\
\text { Type }\end{array}$} & \multicolumn{3}{|c|}{ Coordinates (Angstroms) } \\
\hline & & & $\mathrm{X}$ & $\mathrm{Y}$ & Z \\
\hline 1 & 6 & 0 & -1.140663 & 1.279372 & 0.000995 \\
\hline 2 & 6 & 0 & 0.252651 & 1.251762 & -0.008653 \\
\hline 3 & 6 & 0 & 0.942462 & 0.047299 & -0.005892 \\
\hline 4 & 6 & 0 & 0.201310 & -1.264412 & -0.001768 \\
\hline 5 & 6 & 0 & -1.202797 & -1.219895 & 0.007646 \\
\hline 6 & 6 & 0 & -1.873644 & -0.018885 & 0.009858 \\
\hline 7 & 1 & 0 & -1.689122 & 2.209789 & 0.014599 \\
\hline 8 & 1 & 0 & 0.815723 & 2.181328 & -0.002241 \\
\hline 9 & 1 & 0 & 0.752974 & -2.188776 & -0.113364 \\
\hline 10 & 1 & 0 & -1.764047 & -2.148262 & -0.001800 \\
\hline 11 & 1 & 0 & -2.957558 & -0.006533 & 0.009642 \\
\hline 12 & 7 & 0 & 2.309867 & -0.053131 & -0.043108 \\
\hline 13 & 1 & 0 & 2.863210 & 0.780943 & 0.091202 \\
\hline 14 & 1 & 0 & 2.733829 & -0.908013 & 0.290602 \\
\hline
\end{tabular}




$$
\begin{aligned}
& \mathbf{R}=\mathbf{N}\left(\mathbf{C H}_{\mathbf{3}}\right)_{\mathbf{2}} \mathrm{S}_{0} \\
& \mathrm{E}=-366.3140525 \text { a.u. }
\end{aligned}
$$

\begin{tabular}{|c|c|c|c|c|c|}
\hline \multirow{2}{*}{$\begin{array}{l}\text { Center } \\
\text { Number }\end{array}$} & \multirow{2}{*}{$\begin{array}{l}\text { Atomic } \\
\text { Number }\end{array}$} & \multirow{2}{*}{$\begin{array}{c}\text { Atomic } \\
\text { Type }\end{array}$} & \multicolumn{3}{|c|}{ Coordinates (Angstroms) } \\
\hline & & & $\mathrm{X}$ & Y & Z \\
\hline 1 & 6 & 0 & 0.037327 & -1.942171 & 1.196632 \\
\hline 2 & 6 & 0 & -0.025281 & -0.552644 & 1.206038 \\
\hline 3 & 6 & 0 & -0.072515 & 0.180711 & 0.000000 \\
\hline 4 & 6 & 0 & -0.025281 & -0.552644 & -1.206038 \\
\hline 5 & 6 & 0 & 0.037327 & -1.942171 & -1.196632 \\
\hline 6 & 6 & 0 & 0.066109 & -2.655162 & 0.000000 \\
\hline 7 & 1 & 0 & 0.068794 & -2.470620 & 2.143622 \\
\hline 8 & 1 & 0 & -0.038481 & -0.041089 & 2.158308 \\
\hline 9 & 1 & 0 & -0.038481 & -0.041089 & -2.158308 \\
\hline 10 & 1 & 0 & 0.068794 & -2.470620 & -2.143622 \\
\hline 11 & 1 & 0 & 0.117267 & -3.737278 & 0.000000 \\
\hline 12 & 7 & 0 & -0.176471 & 1.569024 & 0.000000 \\
\hline 13 & 6 & 0 & 0.037327 & 2.295981 & -1.240748 \\
\hline 14 & 1 & 0 & -0.680325 & 1.985951 & -2.004873 \\
\hline 15 & 1 & 0 & -0.119060 & 3.359090 & -1.060040 \\
\hline 16 & 1 & 0 & 1.051061 & 2.160079 & -1.647521 \\
\hline 17 & 6 & 0 & 0.037327 & 2.295981 & 1.240748 \\
\hline 18 & 1 & 0 & -0.119060 & 3.359090 & 1.060040 \\
\hline 19 & 1 & 0 & -0.680325 & 1.985951 & 2.004873 \\
\hline 20 & 1 & 0 & 1.051061 & 2.160079 & 1.647521 \\
\hline
\end{tabular}

$\mathbf{R}=\mathbf{N}\left(\mathbf{C H}_{\mathbf{3}}\right)_{\mathbf{2}} \mathrm{T}_{1}$

\begin{tabular}{|c|c|c|c|c|c|}
\hline \multirow{2}{*}{$\begin{array}{l}\text { Center } \\
\text { Number }\end{array}$} & \multirow{2}{*}{$\begin{array}{l}\text { Atomic } \\
\text { Number }\end{array}$} & \multirow{2}{*}{$\begin{array}{c}\text { Atomic } \\
\text { Type }\end{array}$} & \multicolumn{3}{|c|}{ Coordinates (Angstroms) } \\
\hline & & & $\mathrm{X}$ & $\mathrm{Y}$ & Z \\
\hline 1 & 6 & 0 & 1.895952 & 1.273138 & -0.186217 \\
\hline 2 & 6 & 0 & 0.505168 & 1.229099 & -0.127142 \\
\hline 3 & 6 & 0 & -0.181445 & 0.025341 & 0.037688 \\
\hline 4 & 6 & 0 & 0.596960 & -1.255446 & 0.205738 \\
\hline 5 & 6 & 0 & 2.009430 & -1.180761 & 0.146443 \\
\hline 6 & 6 & 0 & 2.654966 & 0.009794 & -0.053276 \\
\hline 7 & 1 & 0 & 2.417073 & 2.201194 & -0.373807 \\
\hline 8 & 1 & 0 & -0.053337 & 2.146840 & -0.270928 \\
\hline 9 & 1 & 0 & 0.118137 & -2.109435 & 0.668732 \\
\hline 10 & 1 & 0 & 2.586975 & -2.087538 & 0.295104 \\
\hline 11 & 1 & 0 & 3.738010 & 0.039453 & -0.096532 \\
\hline 12 & 7 & 0 & -1.553140 & -0.060780 & 0.153272 \\
\hline 13 & 6 & 0 & -2.274986 & -1.193956 & -0.418383 \\
\hline 14 & 1 & 0 & -1.638855 & -2.075904 & -0.439290 \\
\hline 15 & 1 & 0 & -3.170745 & -1.398790 & 0.172211 \\
\hline 16 & 1 & 0 & -2.578990 & -0.977995 & -1.453300 \\
\hline 17 & 6 & 0 & -2.348983 & 1.145999 & 0.296562 \\
\hline 18 & 1 & 0 & -3.344575 & 0.872363 & 0.650444 \\
\hline 19 & 1 & 0 & -1.885168 & 1.812916 & 1.023083 \\
\hline 20 & 1 & 0 & -2.458932 & 1.683113 & -0.657102 \\
\hline
\end{tabular}

$\mathrm{E}=-366.191025$ a.u. 


$$
\begin{aligned}
& \mathbf{R}=\mathbf{O H ~} \mathrm{S}_{0} \\
& \mathrm{E}=-307.5586319 \text { a.u. }
\end{aligned}
$$

\begin{tabular}{|c|c|c|c|c|c|}
\hline \multirow{2}{*}{$\begin{array}{l}\text { Center } \\
\text { Number }\end{array}$} & \multirow{2}{*}{$\begin{array}{l}\text { Atomic } \\
\text { Number }\end{array}$} & \multirow{2}{*}{$\begin{array}{c}\text { Atomic } \\
\text { Type }\end{array}$} & \multicolumn{3}{|c|}{ Coordinates (Angstroms) } \\
\hline & & & $\mathrm{X}$ & $\mathrm{Y}$ & Z \\
\hline & & & ----------1 & ----------1 & --------- \\
\hline 1 & 6 & 0 & -1.187840 & -1.162180 & 0.000000 \\
\hline 2 & 6 & 0 & -1.204282 & 0.231745 & 0.000000 \\
\hline 3 & 6 & 0 & 0.000000 & 0.938152 & 0.000000 \\
\hline 4 & 6 & 0 & 1.215579 & 0.252752 & 0.000000 \\
\hline 5 & 6 & 0 & 1.218472 & -1.138781 & 0.000000 \\
\hline 6 & 6 & 0 & 0.020797 & -1.855110 & 0.000000 \\
\hline 7 & 1 & 0 & -2.126623 & -1.704651 & 0.000000 \\
\hline 8 & 1 & 0 & -2.148862 & 0.767950 & 0.000000 \\
\hline 9 & 1 & 0 & 2.138691 & 0.819413 & 0.000000 \\
\hline 10 & 1 & 0 & 2.165531 & -1.666804 & 0.000000 \\
\hline 11 & 1 & 0 & 0.030124 & -2.938376 & 0.000000 \\
\hline 12 & 8 & 0 & 0.050909 & 2.307122 & 0.000000 \\
\hline 13 & 1 & 0 & -0.842490 & 2.666024 & 0.000000 \\
\hline
\end{tabular}

\section{$\mathbf{R}=\mathbf{O H} \mathrm{T}_{1}$

\begin{tabular}{|c|c|c|c|c|c|}
\hline \multirow{2}{*}{$\begin{array}{l}\text { Center } \\
\text { Number }\end{array}$} & \multirow{2}{*}{$\begin{array}{l}\text { Atomic } \\
\text { Number }\end{array}$} & \multirow{2}{*}{$\begin{array}{l}\text { Atomic } \\
\text { Type }\end{array}$} & \multicolumn{3}{|c|}{ Coordinates (Angstroms) } \\
\hline & & & $\mathrm{X}$ & Y & Z \\
\hline 1 & 6 & 0 & -1.319910 & -1.062978 & 0.000000 \\
\hline 2 & 6 & 0 & -1.229903 & 0.337547 & 0.000000 \\
\hline 3 & 6 & 0 & 0.000000 & 0.950038 & 0.000000 \\
\hline 4 & 6 & 0 & 1.280097 & 0.158783 & 0.000000 \\
\hline 5 & 6 & 0 & 1.178709 & -1.244107 & 0.000000 \\
\hline 6 & 6 & 0 & -0.044998 & -1.864449 & 0.000000 \\
\hline 7 & 1 & 0 & -2.274592 & -1.567997 & 0.000000 \\
\hline 8 & 1 & 0 & -2.133382 & 0.942190 & 0.000000 \\
\hline 9 & 1 & 0 & 2.219234 & 0.692434 & 0.000000 \\
\hline 10 & 1 & 0 & 2.084988 & -1.840088 & 0.000000 \\
\hline 11 & 1 & 0 & -0.112010 & -2.945638 & 0.000000 \\
\hline 12 & 8 & 0 & 0.208644 & 2.289360 & 0.000000 \\
\hline 13 & 1 & 0 & -0.637360 & 2.755207 & 0.000000 \\
\hline
\end{tabular} \\ $\mathrm{E}=-307.4245929$ a.u.}




\begin{tabular}{|c|c|c|c|c|c|}
\hline $\begin{array}{l}\mathbf{R}=\mathbf{O C H} \\
\mathrm{E}=-346.8\end{array}$ & 8 a.u. & & & & \\
\hline Center & Atomic & Atomic & Coor & inates (Ang & roms ) \\
\hline Number & Number & Type & $\mathrm{X}$ & $Y$ & Z \\
\hline 1 & 6 & 0 & 0.468256 & 1.840225 & 0.000000 \\
\hline 2 & 6 & 0 & 0.925274 & 0.519602 & 0.000000 \\
\hline 3 & 6 & 0 & 0.000000 & -0.527780 & 0.000000 \\
\hline 4 & 6 & 0 & -1.371877 & -0.245894 & 0.000000 \\
\hline 5 & 6 & 0 & -1.809657 & 1.071373 & 0.000000 \\
\hline 6 & 6 & 0 & -0.892394 & 2.125887 & 0.000000 \\
\hline 7 & 1 & 0 & 1.192245 & 2.647592 & 0.000000 \\
\hline 8 & 1 & 0 & 1.989156 & 0.324851 & 0.000000 \\
\hline 9 & 1 & 0 & -2.071031 & -1.073386 & 0.000000 \\
\hline 10 & 1 & 0 & -2.874213 & 1.277553 & 0.000000 \\
\hline 11 & 1 & 0 & -1.237014 & 3.153026 & 0.000000 \\
\hline 12 & 8 & 0 & 0.332799 & -1.852429 & 0.000000 \\
\hline 13 & 6 & 0 & 1.708306 & -2.207905 & 0.000000 \\
\hline 14 & 1 & 0 & 2.218390 & -1.833356 & 0.894440 \\
\hline 15 & 1 & 0 & 1.734237 & -3.296548 & 0.000000 \\
\hline 16 & 1 & 0 & 2.218390 & -1.833356 & -0.894440 \\
\hline
\end{tabular}

\section{$\mathbf{R}=\mathbf{O C H}_{3} \mathrm{~T}_{1}$}

$\mathrm{E}=-346.7360259$ a.u.

\begin{tabular}{|c|c|c|c|c|c|}
\hline \multirow{2}{*}{$\begin{array}{l}\text { Center } \\
\text { Number }\end{array}$} & \multirow{2}{*}{$\begin{array}{l}\text { Atomic } \\
\text { Number }\end{array}$} & \multirow{2}{*}{$\begin{array}{c}\text { Atomic } \\
\text { Type }\end{array}$} & \multicolumn{3}{|c|}{ Coordinates (Angstroms) } \\
\hline & & & $\mathrm{X}$ & $Y$ & Z \\
\hline 1 & 6 & 0 & 0.756297 & 1.770839 & 0.000000 \\
\hline 2 & 6 & 0 & 1.034253 & 0.390829 & 0.000000 \\
\hline 3 & 6 & 0 & 0.000000 & -0.515527 & 0.000000 \\
\hline 4 & 6 & 0 & -1.436697 & -0.067225 & 0.000000 \\
\hline 5 & 6 & 0 & -1.699590 & 1.318121 & 0.000000 \\
\hline 6 & 6 & 0 & -0.675462 & 2.226133 & 0.000000 \\
\hline 7 & 1 & 0 & 1.552057 & 2.501549 & 0.000000 \\
\hline 8 & 1 & 0 & 2.064046 & 0.055990 & 0.000000 \\
\hline 9 & 1 & 0 & -2.208902 & -0.822688 & 0.000000 \\
\hline 10 & 1 & 0 & -2.728346 & 1.662101 & 0.000000 \\
\hline 11 & 1 & 0 & -0.882749 & 3.289535 & 0.000000 \\
\hline 12 & 8 & 0 & 0.099570 & -1.860396 & 0.000000 \\
\hline 13 & 6 & 0 & 1.397624 & -2.450373 & 0.000000 \\
\hline 14 & 1 & 0 & 1.955758 & -2.156806 & 0.894256 \\
\hline 15 & 1 & 0 & 1.237271 & -3.526487 & 0.000000 \\
\hline 16 & 1 & 0 & 1.955758 & -2.156806 & -0.894256 \\
\hline
\end{tabular}




$$
\begin{aligned}
& \mathbf{R}=\mathbf{F ~} \mathrm{S}_{0} \\
& \mathrm{E}=-331.5801119 \text { a.u. }
\end{aligned}
$$

\begin{tabular}{|c|c|c|c|c|c|}
\hline \multirow{2}{*}{$\begin{array}{l}\text { Center } \\
\text { Number }\end{array}$} & \multirow{2}{*}{$\begin{array}{l}\text { Atomic } \\
\text { Number }\end{array}$} & \multirow{2}{*}{$\begin{array}{l}\text { Atomic } \\
\text { Type }\end{array}$} & \multicolumn{3}{|c|}{ Coordinates (Angstroms) } \\
\hline & & & $\mathrm{X}$ & $Y$ & Z \\
\hline 1 & 6 & 0 & 0.000000 & 1.206735 & -1.134261 \\
\hline 2 & 6 & 0 & 0.000000 & 1.215919 & 0.259986 \\
\hline 3 & 6 & 0 & 0.000000 & 0.000000 & 0.925927 \\
\hline 4 & 6 & 0 & 0.000000 & -1.215919 & 0.259986 \\
\hline 5 & 6 & 0 & 0.000000 & -1.206735 & -1.134261 \\
\hline 6 & 6 & 0 & 0.000000 & 0.000000 & -1.833179 \\
\hline 7 & 1 & 0 & 0.000000 & 2.147659 & -1.672595 \\
\hline 8 & 1 & 0 & 0.000000 & 2.138900 & 0.826391 \\
\hline 9 & 1 & 0 & 0.000000 & -2.138900 & 0.826391 \\
\hline 10 & 1 & 0 & 0.000000 & -2.147659 & -1.672595 \\
\hline 11 & 1 & 0 & 0.000000 & 0.000000 & -2.916679 \\
\hline 12 & 9 & 0 & 0.000000 & 0.000000 & 2.282654 \\
\hline
\end{tabular}

\begin{tabular}{|c|c|c|c|c|c|}
\hline \multirow{2}{*}{$\begin{array}{l}\text { Center } \\
\text { Number }\end{array}$} & \multirow{2}{*}{$\begin{array}{l}\text { Atomic } \\
\text { Number }\end{array}$} & \multirow{2}{*}{$\begin{array}{c}\text { Atomic } \\
\text { Type }\end{array}$} & \multicolumn{3}{|c|}{ Coordinates (Angstroms) } \\
\hline & & & $\mathrm{X}$ & Y & Z \\
\hline 1 & 6 & 0 & -1.167409 & 1.242116 & 0.000000 \\
\hline 2 & 6 & 0 & -1.281249 & -0.155858 & 0.000000 \\
\hline 3 & 6 & 0 & 0.000000 & -0.944263 & 0.000000 \\
\hline 4 & 6 & 0 & 1.236890 & -0.371206 & 0.000000 \\
\hline 5 & 6 & 0 & 1.336725 & 1.029609 & 0.000000 \\
\hline 6 & 6 & 0 & 0.063615 & 1.852759 & 0.000000 \\
\hline 7 & 1 & 0 & -2.067980 & 1.846467 & 0.000000 \\
\hline 8 & 1 & 0 & -2.223973 & -0.683166 & 0.000000 \\
\hline 9 & 1 & 0 & 2.118917 & -1.000854 & 0.000000 \\
\hline 10 & 1 & 0 & 2.296211 & 1.525786 & 0.000000 \\
\hline 11 & 1 & 0 & 0.144921 & 2.932316 & 0.000000 \\
\hline 12 & 9 & 0 & -0.155504 & -2.282165 & 0.000000 \\
\hline
\end{tabular}

$$
\begin{aligned}
& \mathbf{R}=\mathbf{F ~ T}_{1} \\
& \mathrm{E}=-331.4402339 \text { a.u. }
\end{aligned}
$$




\begin{tabular}{|c|c|c|c|c|c|}
\hline \multicolumn{6}{|c|}{$\begin{array}{l}\mathbf{R}=\mathbf{S i H}_{3} \mathrm{~S}_{0} \\
\mathrm{E}=-523.0297441 \text { a.u. }\end{array}$} \\
\hline \multirow{2}{*}{$\begin{array}{l}\text { Center } \\
\text { Number }\end{array}$} & \multirow{2}{*}{$\begin{array}{l}\text { Atomic } \\
\text { Number }\end{array}$} & \multirow{2}{*}{$\begin{array}{l}\text { Atomic } \\
\text { Type }\end{array}$} & \multicolumn{3}{|c|}{ Coordinates (Angstroms) } \\
\hline & & & $\mathrm{X}$ & $\mathrm{Y}$ & Z \\
\hline 1 & 6 & 0 & 0.012083 & -1.650886 & -1.205586 \\
\hline 2 & 6 & 0 & 0.012083 & -0.257050 & -1.203262 \\
\hline 3 & 6 & 0 & 0.008307 & 0.466400 & 0.000000 \\
\hline 4 & 6 & 0 & 0.012083 & -0.257050 & 1.203262 \\
\hline 5 & 6 & 0 & 0.012083 & -1.650886 & 1.205586 \\
\hline 6 & 6 & 0 & 0.011423 & -2.350355 & 0.000000 \\
\hline 7 & 1 & 0 & 0.016517 & -2.190148 & -2.146650 \\
\hline 8 & 1 & 0 & 0.019555 & 0.269697 & -2.152758 \\
\hline 9 & 1 & 0 & 0.019555 & 0.269697 & 2.152758 \\
\hline 10 & 1 & 0 & 0.016517 & -2.190148 & 2.146650 \\
\hline 11 & 1 & 0 & 0.014255 & -3.434847 & 0.000000 \\
\hline 12 & 14 & 0 & -0.027421 & 2.348627 & 0.000000 \\
\hline 13 & 1 & 0 & 0.656185 & 2.864914 & -1.214497 \\
\hline 14 & 1 & 0 & -1.423243 & 2.864114 & 0.000000 \\
\hline 15 & 1 & 0 & 0.656185 & 2.864914 & 1.214497 \\
\hline
\end{tabular}

\section{$\mathbf{R}=\mathbf{S i H}_{3} \mathrm{~T}_{1}$}

$\mathrm{E}=-522.8977178$ a.u.

\begin{tabular}{|c|c|c|c|c|c|}
\hline \multirow{2}{*}{$\begin{array}{l}\text { Center } \\
\text { Number }\end{array}$} & \multirow{2}{*}{$\begin{array}{l}\text { Atomic } \\
\text { Number }\end{array}$} & \multirow{2}{*}{$\begin{array}{c}\text { Atomic } \\
\text { Type }\end{array}$} & \multicolumn{3}{|c|}{ Coordinates (Angstroms) } \\
\hline & & & $\mathrm{X}$ & $\mathrm{Y}$ & Z \\
\hline 1 & 6 & 0 & 0.641177 & 1.356728 & 1.242464 \\
\hline 2 & 6 & 0 & 0.641177 & -0.002324 & 1.253854 \\
\hline 3 & 6 & 0 & 0.239160 & -0.690076 & 0.000000 \\
\hline 4 & 6 & 0 & 0.641177 & -0.002324 & -1.253854 \\
\hline 5 & 6 & 0 & 0.641177 & 1.356728 & -1.242464 \\
\hline 6 & 6 & 0 & 0.508913 & 2.068163 & 0.000000 \\
\hline 7 & 1 & 0 & 0.824761 & 1.916634 & 2.154468 \\
\hline 8 & 1 & 0 & 0.854948 & -0.567952 & 2.154654 \\
\hline 9 & 1 & 0 & 0.854948 & -0.567952 & -2.154654 \\
\hline 10 & 1 & 0 & 0.824761 & 1.916634 & -2.154468 \\
\hline 11 & 1 & 0 & 0.512243 & 3.151082 & 0.000000 \\
\hline 12 & 14 & 0 & -1.323237 & -1.733581 & 0.000000 \\
\hline 13 & 1 & 0 & -2.573496 & -0.918981 & 0.000000 \\
\hline 14 & 1 & 0 & -1.324773 & -2.590353 & -1.214932 \\
\hline 15 & 1 & 0 & -1.324773 & -2.590353 & 1.214932 \\
\hline
\end{tabular}




$$
\begin{aligned}
& \mathbf{R}=\mathbf{P H}_{\mathbf{2}} \mathrm{S}_{0} \\
& \mathrm{E}=-574.2803668 \text { a.u. }
\end{aligned}
$$

\begin{tabular}{|c|c|c|c|c|c|}
\hline \multirow{2}{*}{$\begin{array}{l}\text { Center } \\
\text { Number }\end{array}$} & \multirow{2}{*}{$\begin{array}{l}\text { Atomic } \\
\text { Number }\end{array}$} & \multirow{2}{*}{$\begin{array}{c}\text { Atomic } \\
\text { Type }\end{array}$} & \multicolumn{3}{|c|}{ Coordinates (Angstroms) } \\
\hline & & & $\mathrm{X}$ & Y & Z \\
\hline & & & ---------- & --------- & ---------- \\
\hline 1 & 6 & 0 & -0.219185 & 1.203987 & -0.004984 \\
\hline 2 & 6 & 0 & -1.611756 & 1.212325 & 0.004682 \\
\hline 3 & 6 & 0 & -2.320802 & 0.010774 & 0.012423 \\
\hline 4 & 6 & 0 & -1.629886 & -1.197963 & 0.003085 \\
\hline 5 & 6 & 0 & -0.234900 & -1.205249 & -0.016514 \\
\hline 6 & 6 & 0 & 0.487860 & -0.006394 & -0.013014 \\
\hline 7 & 1 & 0 & 0.319516 & 2.145205 & -0.006063 \\
\hline 8 & 1 & 0 & -2.144160 & 2.157174 & 0.008271 \\
\hline 9 & 1 & 0 & -3.404951 & 0.018984 & 0.021354 \\
\hline 10 & 1 & 0 & -2.173410 & -2.136277 & 0.003321 \\
\hline 11 & 1 & 0 & 0.295522 & -2.151435 & -0.039348 \\
\hline 12 & 15 & 0 & 2.340531 & -0.112391 & -0.033847 \\
\hline 13 & 1 & 0 & 2.591448 & 1.138081 & -0.664785 \\
\hline 14 & 1 & 0 & 2.580078 & 0.409256 & 1.270880 \\
\hline
\end{tabular}

\begin{tabular}{|c|c|c|c|c|c|}
\hline \multirow{2}{*}{$\begin{array}{l}\text { Center } \\
\text { Number }\end{array}$} & \multirow{2}{*}{$\begin{array}{l}\text { Atomic } \\
\text { Number }\end{array}$} & \multirow{2}{*}{$\begin{array}{c}\text { Atomic } \\
\text { Type }\end{array}$} & \multicolumn{3}{|c|}{ Coordinates (Angstroms) } \\
\hline & & & $\mathrm{X}$ & $\mathrm{Y}$ & Z \\
\hline 1 & 6 & 0 & 0.246180 & 1.261386 & 0.458497 \\
\hline 2 & 6 & 0 & 1.494242 & 1.237069 & -0.082553 \\
\hline 3 & 6 & 0 & 2.111110 & -0.003128 & -0.449271 \\
\hline 4 & 6 & 0 & 1.490616 & -1.239721 & -0.088330 \\
\hline 5 & 6 & 0 & 0.243283 & -1.260404 & 0.458158 \\
\hline 6 & 6 & 0 & -0.529519 & 0.002418 & 0.489989 \\
\hline 7 & 1 & 0 & -0.188406 & 2.183277 & 0.830624 \\
\hline 8 & 1 & 0 & 2.062559 & 2.157326 & -0.175870 \\
\hline 9 & 1 & 0 & 3.109821 & -0.003820 & -0.867366 \\
\hline 10 & 1 & 0 & 2.054653 & -2.162329 & -0.185563 \\
\hline 11 & 1 & 0 & -0.191112 & -2.179521 & 0.836541 \\
\hline 12 & 15 & 0 & -2.193279 & 0.011227 & -0.193997 \\
\hline 13 & 1 & 0 & -2.108946 & 0.998864 & -1.223967 \\
\hline 14 & 1 & 0 & -2.174855 & -1.147923 & -1.023396 \\
\hline
\end{tabular}

$$
\begin{aligned}
& \mathbf{R}=\mathbf{P H}_{2} \mathrm{~T}_{1} \\
& \mathrm{E}=-574.1538476 \text { a.u. }
\end{aligned}
$$




$$
\begin{aligned}
& \mathbf{R}=\mathbf{S H ~ S}_{0} \\
& \mathrm{E}=-630.5252609 \text { a.u. }
\end{aligned}
$$

\begin{tabular}{|c|c|c|c|c|c|}
\hline \multirow{2}{*}{$\begin{array}{l}\text { Center } \\
\text { Number }\end{array}$} & \multirow{2}{*}{$\begin{array}{l}\text { Atomic } \\
\text { Number }\end{array}$} & \multirow{2}{*}{$\begin{array}{c}\text { Atomic } \\
\text { Type }\end{array}$} & \multicolumn{3}{|c|}{ Coordinates (Angstroms) } \\
\hline & & & $\mathrm{X}$ & $\mathrm{Y}$ & Z \\
\hline & & & ---------- & ---------- & --------- \\
\hline 1 & 6 & 0 & -1.199717 & -1.593436 & 0.000000 \\
\hline 2 & 6 & 0 & -1.207154 & -0.201310 & 0.000000 \\
\hline 3 & 6 & 0 & 0.000000 & 0.506116 & 0.000000 \\
\hline 4 & 6 & 0 & 1.209263 & -0.196227 & 0.000000 \\
\hline 5 & 6 & 0 & 1.205982 & -1.589234 & 0.000000 \\
\hline 6 & 6 & 0 & 0.004685 & -2.295475 & 0.000000 \\
\hline 7 & 1 & 0 & -2.142295 & -2.129392 & 0.000000 \\
\hline 8 & 1 & 0 & -2.150003 & 0.334196 & 0.000000 \\
\hline 9 & 1 & 0 & 2.152638 & 0.338098 & 0.000000 \\
\hline 10 & 1 & 0 & 2.150554 & -2.121873 & 0.000000 \\
\hline 11 & 1 & 0 & 0.007041 & -3.379017 & 0.000000 \\
\hline 12 & 16 & 0 & -0.083832 & 2.291378 & 0.000000 \\
\hline 13 & 1 & 0 & 1.245028 & 2.513343 & 0.000000 \\
\hline
\end{tabular}

\begin{tabular}{|c|c|c|c|c|c|}
\hline \multirow{2}{*}{$\begin{array}{l}\text { Center } \\
\text { Number }\end{array}$} & \multirow{2}{*}{$\begin{array}{l}\text { Atomic } \\
\text { Number }\end{array}$} & \multirow{2}{*}{$\begin{array}{c}\text { Atomic } \\
\text { Type }\end{array}$} & \multicolumn{3}{|c|}{ Coordinates (Angstroms) } \\
\hline & & & $\mathrm{X}$ & Y & Z \\
\hline 1 & 6 & 0 & 1.473075 & -1.221963 & -0.090693 \\
\hline 2 & 6 & 0 & 0.233197 & -1.266936 & 0.465895 \\
\hline 3 & 6 & 0 & -0.556470 & -0.019965 & 0.584030 \\
\hline 4 & 6 & 0 & 0.199011 & 1.251629 & 0.477180 \\
\hline 5 & 6 & 0 & 1.436653 & 1.248693 & -0.097505 \\
\hline 6 & 6 & 0 & 2.076523 & 0.026597 & -0.446304 \\
\hline 7 & 1 & 0 & 2.044001 & -2.136922 & -0.214797 \\
\hline 8 & 1 & 0 & -0.198058 & -2.204477 & 0.801571 \\
\hline 9 & 1 & 0 & -0.249913 & 2.170716 & 0.840450 \\
\hline 10 & 1 & 0 & 1.974028 & 2.183203 & -0.227827 \\
\hline 11 & 1 & 0 & 3.065320 & 0.040855 & -0.887109 \\
\hline 12 & 16 & 0 & -2.087932 & -0.086529 & -0.339526 \\
\hline 13 & 1 & 0 & -2.400403 & 1.222763 & -0.235498 \\
\hline
\end{tabular}

$$
\begin{aligned}
& \mathbf{R}=\mathbf{S H ~ T}_{1} \\
& \mathrm{E}=-630.4034447 \text { a.u. }
\end{aligned}
$$


$\mathbf{R}=\mathbf{C l ~ S}_{0}$

$\mathrm{E}=-691.9342153$ a.u.

\begin{tabular}{|c|c|c|c|c|c|}
\hline \multirow{2}{*}{$\begin{array}{l}\text { Center } \\
\text { Number }\end{array}$} & \multirow{2}{*}{$\begin{array}{l}\text { Atomic } \\
\text { Number }\end{array}$} & \multirow{2}{*}{$\begin{array}{c}\text { Atomic } \\
\text { Type }\end{array}$} & \multicolumn{3}{|c|}{ Coordinates (Angstroms) } \\
\hline & & & $\mathrm{X}$ & $Y$ & Z \\
\hline 1 & 6 & 0 & 0.000000 & 1.205496 & -1.572630 \\
\hline 2 & 6 & 0 & 0.000000 & 1.213602 & -0.178716 \\
\hline 3 & 6 & 0 & 0.000000 & 0.000000 & 0.502261 \\
\hline 4 & 6 & 0 & 0.000000 & -1.213602 & -0.178716 \\
\hline 5 & 6 & 0 & 0.000000 & -1.205496 & -1.572630 \\
\hline 6 & 6 & 0 & 0.000000 & 0.000000 & -2.272306 \\
\hline 7 & 1 & 0 & 0.000000 & 2.147558 & -2.109134 \\
\hline 8 & 1 & 0 & 0.000000 & 2.145223 & 0.372678 \\
\hline 9 & 1 & 0 & 0.000000 & -2.145223 & 0.372678 \\
\hline 10 & 1 & 0 & 0.000000 & -2.147558 & -2.109134 \\
\hline 11 & 1 & 0 & 0.000000 & 0.000000 & -3.355963 \\
\hline 12 & 17 & 0 & 0.000000 & 0.000000 & 2.262665 \\
\hline
\end{tabular}

\section{$\mathbf{R}=\mathbf{C l T}_{1}$ \\ $\mathrm{E}=-691.8085574$ a.u.}

\begin{tabular}{|c|c|c|c|c|c|}
\hline \multirow{2}{*}{$\begin{array}{l}\text { Center } \\
\text { Number }\end{array}$} & \multirow{2}{*}{$\begin{array}{l}\text { Atomic } \\
\text { Number }\end{array}$} & \multirow{2}{*}{$\begin{array}{c}\text { Atomic } \\
\text { Type }\end{array}$} & \multicolumn{3}{|c|}{ Coordinates (Angstroms) } \\
\hline & & & $\mathrm{x}$ & $\mathrm{Y}$ & Z \\
\hline 1 & 6 & 0 & -0.574362 & 1.299462 & 1.236325 \\
\hline 2 & 6 & 0 & -0.574362 & -0.065286 & 1.258301 \\
\hline 3 & 6 & 0 & -0.325286 & -0.784073 & 0.000000 \\
\hline 4 & 6 & 0 & -0.574362 & -0.065286 & -1.258301 \\
\hline 5 & 6 & 0 & -0.574362 & 1.299462 & -1.236325 \\
\hline 6 & 6 & 0 & -0.524220 & 2.004259 & 0.000000 \\
\hline 7 & 1 & 0 & -0.675517 & 1.858886 & 2.160716 \\
\hline 8 & 1 & 0 & -0.688300 & -0.624616 & 2.179580 \\
\hline 9 & 1 & 0 & -0.688300 & -0.624616 & -2.179580 \\
\hline 10 & 1 & 0 & -0.675517 & 1.858886 & -2.160716 \\
\hline 11 & 1 & 0 & -0.543194 & 3.087179 & 0.000000 \\
\hline 12 & 17 & 0 & 1.303091 & -1.628644 & 0.000000 \\
\hline
\end{tabular}




\begin{tabular}{|c|c|c|c|c|c|}
\hline \multicolumn{6}{|c|}{$\begin{array}{l}\mathbf{R}=\mathbf{B r} \mathrm{S}_{0} \\
\mathrm{E}=-2805.8538831 \text { a.u. }\end{array}$} \\
\hline \multirow{2}{*}{$\begin{array}{l}\text { Center } \\
\text { Number }\end{array}$} & \multirow{2}{*}{$\begin{array}{l}\text { Atomic } \\
\text { Number }\end{array}$} & \multirow{2}{*}{$\begin{array}{l}\text { Atomic } \\
\text { Type }\end{array}$} & \multicolumn{3}{|c|}{ Coordinates (Angstroms) } \\
\hline & & & $\mathrm{X}$ & $\mathrm{Y}$ & Z \\
\hline 1 & 6 & 0 & 0.000000 & 1.205469 & -2.181859 \\
\hline 2 & 6 & 0 & 0.000000 & 1.214092 & -0.787413 \\
\hline 3 & 6 & 0 & 0.000000 & 0.000000 & -0.107095 \\
\hline 4 & 6 & 0 & 0.000000 & -1.214092 & -0.787413 \\
\hline 5 & 6 & 0 & 0.000000 & -1.205469 & -2.181859 \\
\hline 6 & 6 & 0 & 0.000000 & 0.000000 & -2.881343 \\
\hline 7 & 1 & 0 & 0.000000 & 2.147714 & -2.718214 \\
\hline 8 & 1 & 0 & 0.000000 & 2.148078 & -0.240320 \\
\hline 9 & 1 & 0 & 0.000000 & -2.148078 & -0.240320 \\
\hline 10 & 1 & 0 & 0.000000 & -2.147714 & -2.718214 \\
\hline 11 & 1 & 0 & 0.000000 & 0.000000 & -3.965062 \\
\hline 12 & 35 & 0 & 0.000000 & 0.000000 & 1.812686 \\
\hline
\end{tabular}

\begin{tabular}{|c|c|c|c|c|c|}
\hline \multicolumn{6}{|c|}{$\begin{array}{l}\mathbf{R}=\mathbf{B r} \mathrm{T}_{1} \\
\mathrm{E}=-2805.7380203 \text { a.u. }\end{array}$} \\
\hline \multirow{2}{*}{$\begin{array}{l}\text { Center } \\
\text { Number }\end{array}$} & \multirow{2}{*}{$\begin{array}{l}\text { Atomic } \\
\text { Number }\end{array}$} & \multirow{2}{*}{$\begin{array}{l}\text { Atomic } \\
\text { Type }\end{array}$} & \multicolumn{3}{|c|}{ Coordinates (Angstroms) } \\
\hline & & & $\mathrm{X}$ & $Y$ & Z \\
\hline 1 & 6 & 0 & -1.152354 & 1.328713 & 0.422490 \\
\hline 2 & 6 & 0 & -0.554901 & 0.286843 & 1.173348 \\
\hline 3 & 6 & 0 & -1.007519 & -0.999633 & 0.915069 \\
\hline 4 & 6 & 0 & -1.960121 & -1.345587 & -0.008640 \\
\hline 5 & 6 & 0 & -2.529304 & -0.293018 & -0.739800 \\
\hline 6 & 6 & 0 & -2.123402 & 1.031840 & -0.520961 \\
\hline 7 & 1 & 0 & -0.838507 & 2.351940 & 0.593194 \\
\hline 8 & 1 & 0 & 0.121947 & 0.505827 & 1.989172 \\
\hline 9 & 1 & 0 & -2.261743 & -2.372769 & -0.176801 \\
\hline 10 & 1 & 0 & -3.286285 & -0.509594 & -1.486161 \\
\hline 11 & 1 & 0 & -2.575089 & 1.830142 & -1.097726 \\
\hline 12 & 35 & 0 & 1.851579 & -0.053157 & -0.207735 \\
\hline
\end{tabular}




$$
\begin{aligned}
& \mathbf{R}=\mathbf{N H}_{\mathbf{3}}{ }^{+} \mathrm{S}_{0} \\
& \mathrm{E}=-288.0344257 \text { a.u. }
\end{aligned}
$$

\begin{tabular}{|c|c|c|c|c|c|}
\hline \multirow{2}{*}{$\begin{array}{l}\text { Center } \\
\text { Number }\end{array}$} & \multirow{2}{*}{$\begin{array}{l}\text { Atomic } \\
\text { Number }\end{array}$} & \multirow{2}{*}{$\begin{array}{c}\text { Atomic } \\
\text { Type }\end{array}$} & \multicolumn{3}{|c|}{ Coordinates (Angstroms) } \\
\hline & & & $\mathrm{X}$ & $\mathrm{Y}$ & $\mathrm{Z}$ \\
\hline 1 & 6 & 0 & 1.211061 & -1.224596 & 0.000000 \\
\hline 2 & 6 & 0 & 1.222147 & 0.168250 & 0.000000 \\
\hline 3 & 6 & 0 & 0.000000 & 0.826284 & 0.000000 \\
\hline 4 & 6 & 0 & -1.219586 & 0.167154 & 0.000000 \\
\hline 5 & 6 & 0 & -1.209849 & -1.227344 & 0.000000 \\
\hline 6 & 6 & 0 & 0.000137 & -1.917675 & 0.000000 \\
\hline 7 & 1 & 0 & 2.149727 & -1.764342 & 0.000000 \\
\hline 8 & 1 & 0 & 2.161734 & 0.710666 & 0.000000 \\
\hline 9 & 1 & 0 & -2.160056 & 0.708723 & 0.000000 \\
\hline 10 & 1 & 0 & -2.148743 & -1.766578 & 0.000000 \\
\hline 11 & 1 & 0 & 0.001078 & -3.000678 & 0.000000 \\
\hline 12 & 7 & 0 & -0.000924 & 2.323390 & 0.000000 \\
\hline 13 & 1 & 0 & 0.469500 & 2.705387 & 0.828367 \\
\hline 14 & 1 & 0 & 0.469500 & 2.705387 & -0.828367 \\
\hline 15 & 1 & 0 & -0.959732 & 2.685267 & 0.000000 \\
\hline
\end{tabular}

\begin{tabular}{|c|c|c|c|c|c|}
\hline \multirow{2}{*}{$\begin{array}{l}\text { Center } \\
\text { Number }\end{array}$} & \multirow{2}{*}{$\begin{array}{l}\text { Atomic } \\
\text { Number }\end{array}$} & \multirow{2}{*}{$\begin{array}{c}\text { Atomic } \\
\text { Type }\end{array}$} & \multicolumn{3}{|c|}{ Coordinates (Angstroms) } \\
\hline & & & $\mathrm{x}$ & $Y$ & Z \\
\hline 1 & 6 & 0 & 0.388097 & 1.031449 & 1.242143 \\
\hline 2 & 6 & 0 & 0.388097 & -0.332322 & 1.268441 \\
\hline 3 & 6 & 0 & 0.099545 & -1.021179 & 0.000000 \\
\hline 4 & 6 & 0 & 0.388097 & -0.332322 & -1.268441 \\
\hline 5 & 6 & 0 & 0.388097 & 1.031449 & -1.242143 \\
\hline 6 & 6 & 0 & 0.325338 & 1.736921 & 0.000000 \\
\hline 7 & 1 & 0 & 0.515096 & 1.592131 & 2.161651 \\
\hline 8 & 1 & 0 & 0.565023 & -0.893930 & 2.179293 \\
\hline 9 & 1 & 0 & 0.565023 & -0.893930 & -2.179293 \\
\hline 10 & 1 & 0 & 0.515096 & 1.592131 & -2.161651 \\
\hline 11 & 1 & 0 & 0.360876 & 2.818910 & 0.000000 \\
\hline 12 & 7 & 0 & -1.338025 & -1.645611 & 0.000000 \\
\hline 13 & 1 & 0 & -1.474832 & -2.230313 & 0.830352 \\
\hline 14 & 1 & 0 & -2.068906 & -0.919378 & 0.000000 \\
\hline 15 & 1 & 0 & -1.474832 & -2.230313 & -0.830352 \\
\hline
\end{tabular}

$$
\begin{aligned}
& \mathbf{R}=\mathbf{N H}_{3}{ }^{+} \mathrm{T}_{1} \\
& \mathrm{E}=-287.9050276 \text { a.u. }
\end{aligned}
$$




\begin{tabular}{|c|c|c|c|c|c|}
\hline $\begin{array}{l}\mathbf{R}=\mathbf{N O}_{2} \\
E=-436 .\end{array}$ & 15 a.u. & & & & \\
\hline Center & Atomic & Atomic & Coor & inates (Ang & roms) \\
\hline Number & Number & Type & $\mathrm{X}$ & $Y$ & Z \\
\hline 1 & 6 & 0 & 0.000000 & 1.210159 & -1.821198 \\
\hline 2 & 6 & 0 & 0.000000 & 1.218752 & -0.429876 \\
\hline 3 & 6 & 0 & 0.000000 & 0.000000 & 0.241543 \\
\hline 4 & 6 & 0 & 0.000000 & -1.218752 & -0.429876 \\
\hline 5 & 6 & 0 & 0.000000 & -1.210159 & -1.821198 \\
\hline 6 & 6 & 0 & 0.000000 & 0.000000 & -2.515492 \\
\hline 7 & 1 & 0 & 0.000000 & 2.148743 & -2.362381 \\
\hline 8 & 1 & 0 & 0.000000 & 2.140336 & 0.135442 \\
\hline 9 & 1 & 0 & 0.000000 & -2.140336 & 0.135442 \\
\hline 10 & 1 & 0 & 0.000000 & -2.148743 & -2.362381 \\
\hline 11 & 1 & 0 & 0.000000 & 0.000000 & -3.599387 \\
\hline 12 & 7 & 0 & 0.000000 & 0.000000 & 1.722275 \\
\hline 13 & 8 & 0 & 0.000000 & -1.084435 & 2.290870 \\
\hline 14 & 8 & 0 & 0.000000 & 1.084435 & 2.290870 \\
\hline
\end{tabular}

\section{$\mathbf{R}=\mathbf{N O}_{\mathbf{2}} \mathrm{T}_{1}$ \\ $\mathrm{E}=-436.7838219$ a.u.}

\begin{tabular}{|c|c|c|c|c|c|}
\hline \multirow{2}{*}{$\begin{array}{l}\text { Center } \\
\text { Number }\end{array}$} & \multirow{2}{*}{$\begin{array}{l}\text { Atomic } \\
\text { Number }\end{array}$} & \multirow{2}{*}{$\begin{array}{c}\text { Atomic } \\
\text { Type }\end{array}$} & \multicolumn{3}{|c|}{ Coordinates (Angstroms) } \\
\hline & & & $\mathrm{x}$ & $\mathrm{Y}$ & Z \\
\hline 1 & 6 & 0 & 0.037502 & -1.839427 & 1.209523 \\
\hline 2 & 6 & 0 & 0.037502 & -0.451112 & 1.223736 \\
\hline 3 & 6 & 0 & 0.032427 & 0.233112 & 0.000000 \\
\hline 4 & 6 & 0 & 0.037502 & -0.451112 & -1.223736 \\
\hline 5 & 6 & 0 & 0.037502 & -1.839427 & -1.209523 \\
\hline 6 & 6 & 0 & 0.038220 & -2.538018 & 0.000000 \\
\hline 7 & 1 & 0 & 0.034887 & -2.380374 & 2.148440 \\
\hline 8 & 1 & 0 & 0.033442 & 0.104949 & 2.152441 \\
\hline 9 & 1 & 0 & 0.033442 & 0.104949 & -2.152441 \\
\hline 10 & 1 & 0 & 0.034887 & -2.380374 & -2.148440 \\
\hline 11 & 1 & 0 & 0.039765 & -3.621400 & 0.000000 \\
\hline 12 & 7 & 0 & 0.078798 & 1.627600 & 0.000000 \\
\hline 13 & 8 & 0 & -0.128247 & 2.380934 & -1.042419 \\
\hline 14 & 8 & 0 & -0.128247 & 2.380934 & 1.042419 \\
\hline
\end{tabular}




\begin{tabular}{|c|c|c|c|c|c|}
\hline \multicolumn{6}{|c|}{$\begin{array}{l}\mathbf{R}=\mathbf{N O S}_{0} \\
\mathrm{E}=-361.6392457 \text { a.u. }\end{array}$} \\
\hline \multirow{2}{*}{$\begin{array}{l}\text { Center } \\
\text { Number }\end{array}$} & \multirow{2}{*}{$\begin{array}{l}\text { Atomic } \\
\text { Number }\end{array}$} & \multirow{2}{*}{$\begin{array}{l}\text { Atomic } \\
\text { Type }\end{array}$} & \multicolumn{3}{|c|}{ Coordinates (Angstroms) } \\
\hline & & & $\mathrm{X}$ & Y & z \\
\hline 1 & 6 & 0 & -0.767640 & -1.679152 & 0.000000 \\
\hline 2 & 6 & 0 & -1.056864 & -0.322920 & 0.000000 \\
\hline 3 & 6 & 0 & 0.000000 & 0.596057 & 0.000000 \\
\hline 4 & 6 & 0 & 1.329430 & 0.169254 & 0.000000 \\
\hline 5 & 6 & 0 & 1.611567 & -1.193856 & 0.000000 \\
\hline 6 & 6 & 0 & 0.563938 & -2.113925 & 0.000000 \\
\hline 7 & 1 & 0 & -1.571761 & -2.406142 & 0.000000 \\
\hline 8 & 1 & 0 & -2.075494 & 0.045412 & 0.000000 \\
\hline 9 & 1 & 0 & 2.115000 & 0.916226 & 0.000000 \\
\hline 10 & 1 & 0 & 2.638875 & -1.538512 & 0.000000 \\
\hline 11 & 1 & 0 & 0.780880 & -3.176303 & 0.000000 \\
\hline 12 & 7 & 0 & -0.183304 & 2.025387 & 0.000000 \\
\hline 13 & 8 & 0 & -1.335870 & 2.406108 & 0.000000 \\
\hline
\end{tabular}

\section{$\mathbf{R}=\mathbf{N O T}_{1}$ \\ $\mathrm{E}=-361.6201979$ a.u.}

\begin{tabular}{|c|c|c|c|c|c|}
\hline \multirow{2}{*}{$\begin{array}{l}\text { Center } \\
\text { Number }\end{array}$} & \multirow{2}{*}{$\begin{array}{l}\text { Atomic } \\
\text { Number }\end{array}$} & \multirow{2}{*}{$\begin{array}{c}\text { Atomic } \\
\text { Type }\end{array}$} & \multicolumn{3}{|c|}{ Coordinates (Angstroms) } \\
\hline & & & $\mathrm{x}$ & Y & Z \\
\hline 1 & 6 & 0 & -0.745151 & -1.698463 & 0.000000 \\
\hline 2 & 6 & 0 & -1.051975 & -0.345190 & 0.000000 \\
\hline 3 & 6 & 0 & 0.000000 & 0.595178 & 0.000000 \\
\hline 4 & 6 & 0 & 1.340888 & 0.166030 & 0.000000 \\
\hline 5 & 6 & 0 & 1.620141 & -1.191611 & 0.000000 \\
\hline 6 & 6 & 0 & 0.583582 & -2.130801 & 0.000000 \\
\hline 7 & 1 & 0 & -1.550787 & -2.424318 & 0.000000 \\
\hline 8 & 1 & 0 & -2.077560 & 0.001850 & 0.000000 \\
\hline 9 & 1 & 0 & 2.132959 & 0.904926 & 0.000000 \\
\hline 10 & 1 & 0 & 2.652052 & -1.523580 & 0.000000 \\
\hline 11 & 1 & 0 & 0.810047 & -3.190309 & 0.000000 \\
\hline 12 & 7 & 0 & -0.253169 & 1.952822 & 0.000000 \\
\hline 13 & 8 & 0 & -1.334930 & 2.523852 & 0.000000 \\
\hline
\end{tabular}




$$
\mathbf{R}=\mathrm{CHOS}_{0}
$$

$\mathrm{E}=-345.6690898$ a.u.

\begin{tabular}{|c|c|c|c|c|c|}
\hline \multirow{2}{*}{$\begin{array}{l}\text { Center } \\
\text { Number }\end{array}$} & \multirow{2}{*}{$\begin{array}{l}\text { Atomic } \\
\text { Number }\end{array}$} & \multirow{2}{*}{$\begin{array}{l}\text { Atomic } \\
\text { Type }\end{array}$} & \multicolumn{3}{|c|}{ Coordinates (Angstroms) } \\
\hline & & & $\mathrm{X}$ & Y & z \\
\hline 1 & 6 & 0 & -1.615745 & -1.229166 & 0.000000 \\
\hline 2 & 6 & 0 & -1.328147 & 0.133134 & 0.000000 \\
\hline 3 & 6 & 0 & 0.000000 & 0.572544 & 0.000000 \\
\hline 4 & 6 & 0 & 1.044087 & -0.362714 & 0.000000 \\
\hline 5 & 6 & 0 & 0.756104 & -1.721117 & 0.000000 \\
\hline 6 & 6 & 0 & -0.573063 & -2.154692 & 0.000000 \\
\hline 7 & 1 & 0 & -2.645021 & -1.568996 & 0.000000 \\
\hline 8 & 1 & 0 & -2.132900 & 0.862091 & 0.000000 \\
\hline 9 & 1 & 0 & 2.066296 & -0.002758 & 0.000000 \\
\hline 10 & 1 & 0 & 1.561635 & -2.446690 & 0.000000 \\
\hline 11 & 1 & 0 & -0.794088 & -3.216346 & 0.000000 \\
\hline 12 & 6 & 0 & 0.286038 & 2.024994 & 0.000000 \\
\hline 13 & 1 & 0 & -0.617190 & 2.671949 & 0.000000 \\
\hline 14 & 8 & 0 & 1.393202 & 2.515357 & 0.000000 \\
\hline
\end{tabular}

\section{$\mathbf{R}=\mathbf{C H O}_{1}$}

\section{$\mathrm{E}=-345.5603218$ a.u.}

\begin{tabular}{|c|c|c|c|c|c|}
\hline \multirow{2}{*}{$\begin{array}{l}\text { Center } \\
\text { Number }\end{array}$} & \multirow{2}{*}{$\begin{array}{l}\text { Atomic } \\
\text { Number }\end{array}$} & \multirow{2}{*}{$\begin{array}{c}\text { Atomic } \\
\text { Type }\end{array}$} & \multicolumn{3}{|c|}{ Coordinates (Angstroms) } \\
\hline & & & $\mathrm{X}$ & $\mathrm{Y}$ & Z \\
\hline 1 & 6 & 0 & -1.635242 & -1.206695 & 0.000000 \\
\hline 2 & 6 & 0 & -1.348913 & 0.146431 & 0.000000 \\
\hline 3 & 6 & 0 & 0.000000 & 0.596510 & 0.000000 \\
\hline 4 & 6 & 0 & 1.037105 & -0.377167 & 0.000000 \\
\hline 5 & 6 & 0 & 0.725126 & -1.726867 & 0.000000 \\
\hline 6 & 6 & 0 & -0.605819 & -2.157635 & 0.000000 \\
\hline 7 & 1 & 0 & -2.670055 & -1.531163 & 0.000000 \\
\hline 8 & 1 & 0 & -2.153855 & 0.873391 & 0.000000 \\
\hline 9 & 1 & 0 & 2.072062 & -0.055765 & 0.000000 \\
\hline 10 & 1 & 0 & 1.527830 & -2.456292 & 0.000000 \\
\hline 11 & 1 & 0 & -0.838705 & -3.215530 & 0.000000 \\
\hline 12 & 6 & 0 & 0.281078 & 1.984408 & 0.000000 \\
\hline 13 & 1 & 0 & -0.507361 & 2.742382 & 0.000000 \\
\hline 14 & 8 & 0 & 1.481259 & 2.511134 & 0.000000 \\
\hline
\end{tabular}




$$
\mathbf{R}=\mathbf{C O C H}_{3} \mathrm{~S}_{0}
$$

$\mathrm{E}=-385.0016932$ a.u.

\begin{tabular}{|c|c|c|c|c|c|}
\hline \multirow{2}{*}{$\begin{array}{l}\text { Center } \\
\text { Number }\end{array}$} & \multirow{2}{*}{$\begin{array}{l}\text { Atomic } \\
\text { Number }\end{array}$} & \multirow{2}{*}{$\begin{array}{c}\text { Atomic } \\
\text { Type }\end{array}$} & \multicolumn{3}{|c|}{ Coordinates (Angstroms) } \\
\hline & & & $\mathrm{X}$ & Y & Z \\
\hline 1 & 6 & 0 & -1.820999 & 1.276453 & 0.000008 \\
\hline 2 & 6 & 0 & -0.430565 & 1.191352 & 0.000001 \\
\hline 3 & 6 & 0 & 0.204410 & -0.057723 & -0.000012 \\
\hline 4 & 6 & 0 & -0.579987 & -1.219776 & -0.000012 \\
\hline 5 & 6 & 0 & -1.966657 & -1.134713 & -0.000002 \\
\hline 6 & 6 & 0 & -2.590384 & 0.114520 & 0.000007 \\
\hline 7 & 1 & 0 & -2.302780 & 2.247606 & 0.000015 \\
\hline 8 & 1 & 0 & 0.153979 & 2.103331 & 0.000001 \\
\hline 9 & 1 & 0 & -0.077372 & -2.179248 & -0.000022 \\
\hline 10 & 1 & 0 & -2.564298 & -2.039311 & -0.000004 \\
\hline 11 & 1 & 0 & -3.672703 & 0.181292 & 0.000015 \\
\hline 12 & 6 & 0 & 1.698812 & -0.204390 & -0.000028 \\
\hline 13 & 6 & 0 & 2.558587 & 1.046308 & -0.000002 \\
\hline 14 & 1 & 0 & 3.606030 & 0.749662 & 0.000044 \\
\hline 15 & 1 & 0 & 2.355431 & 1.660481 & -0.882101 \\
\hline 16 & 1 & 0 & 2.355358 & 1.660500 & 0.882067 \\
\hline 17 & 8 & 0 & 2.213383 & -1.307063 & 0.000029 \\
\hline
\end{tabular}

\section{$\mathbf{R}=\mathbf{C O C H}_{3} \mathrm{~T}_{1}$} $\mathrm{E}=-384.8910246$ a.u.

\begin{tabular}{|c|c|c|c|c|c|}
\hline \multirow{2}{*}{$\begin{array}{l}\text { Center } \\
\text { Number }\end{array}$} & \multirow{2}{*}{$\begin{array}{l}\text { Atomic } \\
\text { Number }\end{array}$} & \multirow{2}{*}{$\begin{array}{c}\text { Atomic } \\
\text { Type }\end{array}$} & \multicolumn{3}{|c|}{ Coordinates (Angstroms) } \\
\hline & & & $\mathrm{x}$ & $Y$ & Z \\
\hline 1 & 6 & 0 & 0.751777 & -2.096441 & 0.000000 \\
\hline 2 & 6 & 0 & 1.045506 & -0.742404 & 0.000000 \\
\hline 3 & 6 & 0 & 0.000000 & 0.222537 & 0.000000 \\
\hline 4 & 6 & 0 & -1.344573 & -0.249857 & 0.000000 \\
\hline 5 & 6 & 0 & -1.613004 & -1.607050 & 0.000000 \\
\hline 6 & 6 & 0 & -0.573688 & -2.545727 & 0.000000 \\
\hline 7 & 1 & 0 & 1.564821 & -2.814463 & 0.000000 \\
\hline 8 & 1 & 0 & 2.080281 & -0.421591 & 0.000000 \\
\hline 9 & 1 & 0 & -2.159005 & 0.465590 & 0.000000 \\
\hline 10 & 1 & 0 & -2.643966 & -1.944356 & 0.000000 \\
\hline 11 & 1 & 0 & -0.792631 & -3.606699 & 0.000000 \\
\hline 12 & 6 & 0 & 0.285195 & 1.610598 & 0.000000 \\
\hline 13 & 6 & 0 & 1.664403 & 2.239254 & 0.000000 \\
\hline 14 & 1 & 0 & 1.591049 & 3.327252 & 0.000000 \\
\hline 15 & 1 & 0 & 2.219684 & 1.932275 & 0.892124 \\
\hline 16 & 1 & 0 & 2.219684 & 1.932275 & -0.892124 \\
\hline 17 & 8 & 0 & -0.671701 & 2.518032 & 0.000000 \\
\hline
\end{tabular}


$\mathbf{R}=\mathbf{C O O H ~ \mathrm { S } _ { 0 }}$

$\mathrm{E}=-420.9481337$ a.u.

\begin{tabular}{|c|c|c|c|c|c|}
\hline \multirow{2}{*}{$\begin{array}{l}\text { Center } \\
\text { Number }\end{array}$} & \multirow{2}{*}{$\begin{array}{l}\text { Atomic } \\
\text { Number }\end{array}$} & \multirow{2}{*}{$\begin{array}{c}\text { Atomic } \\
\text { Type }\end{array}$} & \multicolumn{3}{|c|}{ Coordinates (Angstroms) } \\
\hline & & & $\mathrm{X}$ & $\mathrm{Y}$ & Z \\
\hline 1 & 6 & 0 & -0.963454 & -1.993959 & 0.000000 \\
\hline 2 & 6 & 0 & -1.125200 & -0.611661 & 0.000000 \\
\hline 3 & 6 & 0 & 0.000000 & 0.221681 & 0.000000 \\
\hline 4 & 6 & 0 & 1.282741 & -0.338202 & 0.000000 \\
\hline 5 & 6 & 0 & 1.438883 & -1.719657 & 0.000000 \\
\hline 6 & 6 & 0 & 0.316241 & -2.548520 & 0.000000 \\
\hline 7 & 1 & 0 & -1.834786 & -2.638719 & 0.000000 \\
\hline 8 & 1 & 0 & -2.115112 & -0.174660 & 0.000000 \\
\hline 9 & 1 & 0 & 2.140702 & 0.322717 & 0.000000 \\
\hline 10 & 1 & 0 & 2.433189 & -2.151168 & 0.000000 \\
\hline 11 & 1 & 0 & 0.438888 & -3.625925 & 0.000000 \\
\hline 12 & 6 & 0 & -0.115802 & 1.703570 & 0.000000 \\
\hline 13 & 8 & 0 & 0.816785 & 2.473291 & 0.000000 \\
\hline 14 & 8 & 0 & -1.403466 & 2.137131 & 0.000000 \\
\hline 15 & 1 & 0 & -1.369899 & 3.104862 & 0.000000 \\
\hline
\end{tabular}

\section{$\mathbf{R}=\mathbf{C O O H ~ T} \mathrm{T}_{1}$}

$\mathrm{E}=-420.8228363$ a.u.

\begin{tabular}{|c|c|c|c|c|c|}
\hline \multirow{2}{*}{$\begin{array}{l}\text { Center } \\
\text { Number }\end{array}$} & \multirow{2}{*}{$\begin{array}{l}\text { Atomic } \\
\text { Number }\end{array}$} & \multirow{2}{*}{$\begin{array}{c}\text { Atomic } \\
\text { Type }\end{array}$} & \multicolumn{3}{|c|}{ Coordinates (Angstroms) } \\
\hline & & & $\mathrm{x}$ & $\mathrm{Y}$ & Z \\
\hline 1 & 6 & 0 & -1.177087 & -1.873182 & 0.000000 \\
\hline 2 & 6 & 0 & -1.234657 & -0.525604 & 0.000000 \\
\hline 3 & 6 & 0 & 0.000000 & 0.257704 & 0.000000 \\
\hline 4 & 6 & 0 & 1.304697 & -0.473213 & 0.000000 \\
\hline 5 & 6 & 0 & 1.325451 & -1.827580 & 0.000000 \\
\hline 6 & 6 & 0 & 0.109521 & -2.582547 & 0.000000 \\
\hline 7 & 1 & 0 & -2.089916 & -2.458117 & 0.000000 \\
\hline 8 & 1 & 0 & -2.179358 & 0.000664 & 0.000000 \\
\hline 9 & 1 & 0 & 2.206151 & 0.123760 & 0.000000 \\
\hline 10 & 1 & 0 & 2.272906 & -2.354714 & 0.000000 \\
\hline 11 & 1 & 0 & 0.128006 & -3.664097 & 0.000000 \\
\hline 12 & 6 & 0 & 0.021332 & 1.690292 & 0.000000 \\
\hline 13 & 8 & 0 & 1.049272 & 2.367993 & 0.000000 \\
\hline 14 & 8 & 0 & -1.219576 & 2.273093 & 0.000000 \\
\hline 15 & 1 & 0 & -1.070904 & 3.228607 & 0.000000 \\
\hline
\end{tabular}




\section{$\mathbf{R}=\mathbf{C O O C H}_{3} \mathrm{~S}_{0}$}

$\mathrm{E}=-460.2581086$ a.u.

\begin{tabular}{|c|c|c|c|c|c|}
\hline \multirow{2}{*}{$\begin{array}{l}\text { Center } \\
\text { Number }\end{array}$} & \multirow{2}{*}{$\begin{array}{l}\text { Atomic } \\
\text { Number }\end{array}$} & \multirow{2}{*}{$\begin{array}{c}\text { Atomic } \\
\text { Type }\end{array}$} & \multicolumn{3}{|c|}{ Coordinates (Angstroms) } \\
\hline & & & $\mathrm{X}$ & $Y$ & Z \\
\hline 1 & 6 & 0 & 2.212201 & 1.236074 & 0.000000 \\
\hline 2 & 6 & 0 & 1.391701 & 0.111590 & 0.000000 \\
\hline 3 & 6 & 0 & 0.000000 & 0.262539 & 0.000000 \\
\hline 4 & 6 & 0 & -0.559670 & 1.545290 & 0.000000 \\
\hline 5 & 6 & 0 & 0.263783 & 2.665951 & 0.000000 \\
\hline 6 & 6 & 0 & 1.650554 & 2.512705 & 0.000000 \\
\hline 7 & 1 & 0 & 3.289684 & 1.117030 & 0.000000 \\
\hline 8 & 1 & 0 & 1.820711 & -0.881602 & 0.000000 \\
\hline 9 & 1 & 0 & -1.638254 & 1.642857 & 0.000000 \\
\hline 10 & 1 & 0 & -0.173433 & 3.657840 & 0.000000 \\
\hline 11 & 1 & 0 & 2.292477 & 3.386670 & 0.000000 \\
\hline 12 & 6 & 0 & -0.928278 & -0.904103 & 0.000000 \\
\hline 13 & 8 & 0 & -2.135587 & -0.820921 & 0.000000 \\
\hline 14 & 8 & 0 & -0.273805 & -2.086552 & 0.000000 \\
\hline 15 & 6 & 0 & -1.102640 & -3.262258 & 0.000000 \\
\hline 16 & 1 & 0 & -0.412493 & -4.103176 & 0.000000 \\
\hline 17 & 1 & 0 & -1.734738 & -3.283278 & 0.888808 \\
\hline 18 & 1 & 0 & -1.734738 & -3.283278 & -0.888808 \\
\hline
\end{tabular}

\section{$\mathbf{R}=\mathbf{C O O C H}_{3} \mathrm{~T}_{1}$}

$\mathrm{E}=-460.1321388$ a.u.

\begin{tabular}{|c|c|c|c|c|c|}
\hline \multirow{2}{*}{$\begin{array}{l}\text { Center } \\
\text { Number }\end{array}$} & \multirow{2}{*}{$\begin{array}{l}\text { Atomic } \\
\text { Number }\end{array}$} & \multirow{2}{*}{$\begin{array}{l}\text { Atomic } \\
\text { Type }\end{array}$} & \multicolumn{3}{|c|}{ Coordinates (Angstroms) } \\
\hline & & & $\mathrm{x}$ & $\mathrm{Y}$ & Z \\
\hline 1 & 6 & 0 & 2.089829 & -1.423515 & 0.000000 \\
\hline 2 & 6 & 0 & 0.753698 & -1.245148 & 0.000000 \\
\hline 3 & 6 & 0 & 0.193733 & 0.113250 & 0.000000 \\
\hline 4 & 6 & 0 & 1.139714 & 1.267111 & 0.000000 \\
\hline 5 & 6 & 0 & 2.474227 & 1.052124 & 0.000000 \\
\hline 6 & 6 & 0 & 3.009068 & -0.283289 & 0.000000 \\
\hline 7 & 1 & 0 & 2.506274 & -2.424568 & 0.000000 \\
\hline 8 & 1 & 0 & 0.069145 & -2.081882 & 0.000001 \\
\hline 9 & 1 & 0 & 0.709892 & 2.259324 & 0.000001 \\
\hline 10 & 1 & 0 & 3.159728 & 1.892205 & 0.000000 \\
\hline 11 & 1 & 0 & 4.077875 & -0.450179 & -0.000001 \\
\hline 12 & 6 & 0 & -1.216964 & 0.386743 & 0.000000 \\
\hline 13 & 8 & 0 & -1.699852 & 1.517872 & 0.000000 \\
\hline 14 & 8 & 0 & -1.986616 & -0.742904 & 0.000000 \\
\hline 15 & 6 & 0 & -3.403686 & -0.524554 & 0.000000 \\
\hline 16 & 1 & 0 & -3.708457 & 0.032670 & -0.887993 \\
\hline 17 & 1 & 0 & -3.851977 & -1.516320 & 0.000000 \\
\hline 18 & 1 & 0 & -3.708457 & 0.032670 & 0.887993 \\
\hline
\end{tabular}




\section{$\mathbf{R}=\mathbf{C O N H}_{2} \mathrm{~S}_{0}$}

$\mathrm{E}=-401.0702583$ a.u.

\begin{tabular}{|c|c|c|c|c|c|}
\hline \multirow{2}{*}{$\begin{array}{l}\text { Center } \\
\text { Number }\end{array}$} & \multirow{2}{*}{$\begin{array}{l}\text { Atomic } \\
\text { Number }\end{array}$} & \multirow{2}{*}{$\begin{array}{c}\text { Atomic } \\
\text { Type }\end{array}$} & \multicolumn{3}{|c|}{ Coordinates (Angstroms) } \\
\hline & & & $\mathrm{X}$ & Y & Z \\
\hline 1 & 6 & 0 & -1.851513 & 1.226514 & -0.139867 \\
\hline 2 & 6 & 0 & -0.458865 & 1.191823 & -0.157200 \\
\hline 3 & 6 & 0 & 0.219613 & -0.024668 & -0.016674 \\
\hline 4 & 6 & 0 & -0.516493 & -1.205598 & 0.127328 \\
\hline 5 & 6 & 0 & -1.906615 & -1.168596 & 0.154659 \\
\hline 6 & 6 & 0 & -2.577143 & 0.047675 & 0.022347 \\
\hline 7 & 1 & 0 & -2.368843 & 2.171376 & -0.261727 \\
\hline 8 & 1 & 0 & 0.090520 & 2.112949 & -0.316263 \\
\hline 9 & 1 & 0 & 0.021401 & -2.141581 & 0.213754 \\
\hline 10 & 1 & 0 & -2.468457 & -2.087882 & 0.275062 \\
\hline 11 & 1 & 0 & -3.660889 & 0.075834 & 0.038136 \\
\hline 12 & 6 & 0 & 1.718702 & -0.136704 & -0.037506 \\
\hline 13 & 8 & 0 & 2.285057 & -1.181248 & -0.314795 \\
\hline 14 & 7 & 0 & 2.419032 & 1.007921 & 0.252917 \\
\hline 15 & 1 & 0 & 1.986744 & 1.771662 & 0.745851 \\
\hline 16 & 1 & 0 & 3.419726 & 0.909504 & 0.334604 \\
\hline
\end{tabular}

\section{$\mathbf{R}=\mathbf{C O N H}_{\mathbf{2}} \mathrm{T}_{1}$}

$\mathrm{E}=-400.9435197$ a.u.

\begin{tabular}{|c|c|c|c|c|c|}
\hline \multirow{2}{*}{$\begin{array}{l}\text { Center } \\
\text { Number }\end{array}$} & \multirow{2}{*}{$\begin{array}{l}\text { Atomic } \\
\text { Number }\end{array}$} & \multirow{2}{*}{$\begin{array}{l}\text { Atomic } \\
\text { Type }\end{array}$} & \multicolumn{3}{|c|}{ Coordinates (Angstroms) } \\
\hline & & & $\mathrm{X}$ & $\mathrm{Y}$ & Z \\
\hline 1 & 6 & 0 & -1.803203 & 1.279604 & -0.063535 \\
\hline 2 & 6 & 0 & -0.475268 & 1.239643 & -0.307774 \\
\hline 3 & 6 & 0 & 0.253893 & -0.038519 & -0.155756 \\
\hline 4 & 6 & 0 & -0.552085 & -1.284666 & -0.121754 \\
\hline 5 & 6 & 0 & -1.879753 & -1.211847 & 0.117421 \\
\hline 6 & 6 & 0 & -2.540564 & 0.067006 & 0.239825 \\
\hline 7 & 1 & 0 & -2.345174 & 2.216549 & -0.135462 \\
\hline 8 & 1 & 0 & 0.063758 & 2.128149 & -0.614366 \\
\hline 9 & 1 & 0 & -0.028219 & -2.223552 & -0.238117 \\
\hline 10 & 1 & 0 & -2.470683 & -2.117247 & 0.199892 \\
\hline 11 & 1 & 0 & -3.599369 & 0.117670 & 0.457032 \\
\hline 12 & 6 & 0 & 1.685410 & -0.129625 & 0.021234 \\
\hline 13 & 8 & 0 & 2.274879 & -1.217165 & -0.083536 \\
\hline 14 & 7 & 0 & 2.392975 & 1.049801 & 0.213057 \\
\hline 15 & 1 & 0 & 1.935881 & 1.822862 & 0.673261 \\
\hline 16 & 1 & 0 & 3.363373 & 0.914703 & 0.456676 \\
\hline
\end{tabular}




$$
\begin{aligned}
& \mathbf{R}=\mathbf{C N ~ S}_{0} \\
& \mathrm{E}=-324.5777612 \text { a.u. }
\end{aligned}
$$

\begin{tabular}{|c|c|c|c|c|c|}
\hline \multirow{2}{*}{$\begin{array}{l}\text { Center } \\
\text { Number }\end{array}$} & \multirow{2}{*}{$\begin{array}{l}\text { Atomic } \\
\text { Number }\end{array}$} & \multirow{2}{*}{$\begin{array}{c}\text { Atomic } \\
\text { Type }\end{array}$} & \multicolumn{3}{|c|}{ Coordinates (Angstroms) } \\
\hline & & & $\mathrm{X}$ & $\mathrm{Y}$ & Z \\
\hline 1 & 6 & 0 & 0.000000 & -1.208739 & -1.480787 \\
\hline 2 & 6 & 0 & 0.000000 & -1.215198 & -0.090261 \\
\hline 3 & 6 & 0 & 0.000000 & 0.000000 & 0.610288 \\
\hline 4 & 6 & 0 & 0.000000 & 1.215198 & -0.090261 \\
\hline 5 & 6 & 0 & 0.000000 & 1.208739 & -1.480787 \\
\hline 6 & 6 & 0 & 0.000000 & 0.000000 & -2.176538 \\
\hline 7 & 1 & 0 & 0.000000 & -2.147736 & -2.021623 \\
\hline 8 & 1 & 0 & 0.000000 & -2.149097 & 0.457900 \\
\hline 9 & 1 & 0 & 0.000000 & 2.149097 & 0.457900 \\
\hline 10 & 1 & 0 & 0.000000 & 2.147736 & -2.021623 \\
\hline 11 & 1 & 0 & 0.000000 & 0.000000 & -3.260446 \\
\hline 12 & 6 & 0 & 0.000000 & 0.000000 & 2.042172 \\
\hline 13 & 7 & 0 & 0.000000 & 0.000000 & 3.197850 \\
\hline
\end{tabular}

\section{$\mathbf{R}=\mathbf{C N ~ T _ { 1 }}$

\begin{tabular}{|c|c|c|c|c|c|}
\hline \multirow{2}{*}{$\begin{array}{l}\text { Center } \\
\text { Number }\end{array}$} & \multirow{2}{*}{$\begin{array}{l}\text { Atomic } \\
\text { Number }\end{array}$} & \multirow{2}{*}{$\begin{array}{c}\text { Atomic } \\
\text { Type }\end{array}$} & \multicolumn{3}{|c|}{ Coordinates (Angstroms) } \\
\hline & & & $\mathrm{x}$ & $\mathrm{Y}$ & Z \\
\hline 1 & 6 & 0 & 0.000000 & -1.251278 & -1.460954 \\
\hline 2 & 6 & 0 & 0.000000 & -1.276992 & -0.112992 \\
\hline 3 & 6 & 0 & 0.000000 & 0.000000 & 0.651739 \\
\hline 4 & 6 & 0 & 0.000000 & 1.276992 & -0.112992 \\
\hline 5 & 6 & 0 & 0.000000 & 1.251278 & -1.460954 \\
\hline 6 & 6 & 0 & 0.000000 & 0.000000 & -2.190633 \\
\hline 7 & 1 & 0 & 0.000000 & -2.181198 & -2.018217 \\
\hline 8 & 1 & 0 & 0.000000 & -2.206109 & 0.441961 \\
\hline 9 & 1 & 0 & 0.000000 & 2.206109 & 0.441961 \\
\hline 10 & 1 & 0 & 0.000000 & 2.181198 & -2.018217 \\
\hline 11 & 1 & 0 & 0.000000 & 0.000000 & -3.272080 \\
\hline 12 & 6 & 0 & 0.000000 & 0.000000 & 2.022817 \\
\hline 13 & 7 & 0 & 0.000000 & 0.000000 & 3.201200 \\
\hline
\end{tabular} \\ $\mathrm{E}=-324.4545876$ a.u.}

